\title{
Mars Mineralogical Spectrometer (MMS) on the Tianwen-1 Mission
}

\author{
Zhiping $\mathrm{He}^{1} \cdot$ Rui $\mathrm{Xu}^{1} \cdot$ Chunlai $\mathrm{Li}^{1} \cdot$ Liyin Yuan ${ }^{1}$ Chengyu Liu ${ }^{1} \cdot$ Gang Lv $^{1}$. \\ Jian Jin ${ }^{1} \cdot$ Jianan Xie $^{1}$ - Chuifeng Kong ${ }^{1} \cdot$ Feifei $\mathrm{Li}^{1} \cdot$ Xiaowen Chen ${ }^{1} \cdot$ Rong Wang $^{1}$. \\ Sheng Xu${ }^{1}$ - Wei Pan ${ }^{1}$. Jincai Wu ${ }^{1}$ Changkun $\mathrm{Li}^{1}$ - Tianhong Wang ${ }^{1}$ Haijun Jin ${ }^{1}$. \\ Hourui Chen ${ }^{1} \cdot$ Jun Qiu $^{1} \cdot$ Jianyu Wang ${ }^{1}$
}

Received: 28 July 2020 / Accepted: 7 February 2021 / Published online: 17 February 2021

(C) The Author(s) 2021

\begin{abstract}
The Mars Mineralogical Spectrometer (MMS) is a hyperspectral imager onboard the Mars orbiter of Tianwen-1, China's first Mars exploration mission. MMS consists of 4 subassemblies: an Optical Sensor Unit (OSU), an Electronics Unit (EU), a Calibration Unit (CU), and a Thermal Control Accessories (TCA). With a $0.5 \mathrm{mrad}$ IFOV and a 416sample cell array for nadir observation, MMS can map the spectral and spatial information of the Martian surface through push-broom scanning, and it can transmit scientific data by hyperspectral mode or multispectral imaging mode through spatial and spectral combination. MMS can perform multi-sample hyperspectral imaging at full spectral resolution $(0.379-1.076 \mu \mathrm{m}$ with $2.73 \mathrm{~nm} / \mathrm{band}, 1.033-3.425 \mu \mathrm{m}$ at $7.5 \mathrm{~nm} / \mathrm{band}$, both spectral ranges at $2.1 \mathrm{~km} /$ pixel at $265 \mathrm{~km}$ ). For the wavelength region of interest, the multispectral mapping mode provides additional options, a subset of 72 bands that are binned to minimum pixel footprints of $265 \mathrm{~m} /$ pixel. The major objective of the MMS is to analyze the compositions and distributions of the minerals on Martian surface, in order to characterize its evolution.
\end{abstract}

Keywords Mars Mineralogical Spectrometer · MMS · Mars · Orbiter · Tianwen-1
Abbreviations
AU Astronomical Unit
CAS Chinese Academy of Sciences
CCD Charge Coupled Devices
DN Digital Number
EQM Engineering Qualification Model
FM Flight Model

The Huoxing-1 (HX-1) / Tianwen-1 (TW-1) mission to Mars

Edited by Chunlai Li and Jianjun Liu

Z. He

hzping@mail.sitp.ac.cn

J. Wang

jywang@mail.sitp.ac.cn

1 Key Laboratory of Space Active Opto-electronics Technology, Shanghai Institute of Technical Physics, Chinese Academy of Sciences, Shanghai, China 


$\begin{array}{ll}\text { FOV } & \text { Field of View } \\ \text { FP } & \text { Focal Panel } \\ \text { FPA } & \text { Focal Panel Array } \\ \text { FPGA } & \text { Field Programmable Gate Array } \\ \text { FWHM } & \text { Full Width at Half Maximum } \\ \text { IFOV } & \text { Instantaneous Field of View } \\ \text { IR } & \text { Infrared } \\ \text { MCT } & \text { Mercury Cadmium Telluride } \\ \text { MLI } & \text { Multilayer Insulation } \\ \text { MMS } & \text { Mars Mineralogical Spectrometer } \\ \text { MTF } & \text { Modular Transfer Function } \\ \text { NA } & \text { Numerical Aperture } \\ \text { NAO } & \text { National Astronomical Observatories } \\ \text { N-MIR } & \text { Near-infrared and Mid-infrared } \\ \text { OSF } & \text { Order-Sorting Filter } \\ \text { TW } & \text { Tianwen } \\ \text { PI } & \text { Principal Investigator } \\ \text { REFF } & \text { Reflectance Factor } \\ \text { RMS } & \text { Root Mean Square } \\ \text { RMSE } & \text { Root Mean Square Error } \\ \text { SITP } & \text { Shanghai Institute of Technical Physics } \\ \text { S/N } & \text { Signal to Noise rate } \\ \text { V-NIR } & \text { Visible and Near-Infrared } \\ & \end{array}$

\section{Introduction}

The first Mars exploration mission of China, Tianwen-1 (TW-1), was successfully launched onboard the Long March 5 carrier rocket from Wenchang Satellite Launch Centre (WSLC) on July 23, 2020. The goals of the mission can be summarised as 'orbiting, landing and roving' (Ye et al. 2017). The spacecraft consists of an orbiter and a descent module, which comprises a heatshield, a backshell, a landing platform, and a rover (Dong et al. 2017). Global and general exploration of Mars will be performed using in-orbit exploration, whereas detailed investigations of key site with high accuracy and resolution will be conducted via roving exploration. The orbiter is expected to begin a remote sensing scientific exploration mission in the summer of 2021 for the next one Martian year, after a successful launch.

The primary scientific objectives of Tianwen-1's mission include: (1) investigating the characteristics of Mar's morphology and its geological structure, (2) investigating the soil characteristics and water-ice distribution on the Martian surface, (3) investigating the material composition on the Martian surface, (4) investigating Mar's ionosphere and the characteristics of its surface climate and environment, (5) investigating the physical field and internal structure of Mars, and (6) detecting and measuring Martian magnetic field characteristics (Ye et al. 2017; Li et al. 2018).

The Mars Mineralogical Spectrometer (MMS) is a remote sensing payload onboard Tianwen-1. Apart from MMS, the orbiter is equipped with High Resolution Imaging Camera (HiRIC), Moderate Resolution Imaging Camera (MoRIC), Mars Orbiter Magnetometer (MOMAG), Mars Ion and Neutral Particle Analyzer (MINPA), Mars Energetic Particles Analyzer (MEPA), and Mars Orbiter Scientific Investigation Radar (MOSIR) (Li et al. 2018; Jia et al. 2018). MMS serves the scientific mission by: (1) obtaining spectral information 
Table 1 Orbital parameters of the remote sensing phase

\begin{tabular}{ll}
\hline Parameter & Designed value \\
\hline Orbit Epoch & 26 Jul 2021 19:45:54.537 \\
Semi-major Axis (km) & 9476.295 \\
Eccentricity & 0.611999 \\
Inclination (deg) & 86.900 \\
RAAN (deg) & 106.189 \\
Argument of Perigee (deg) & 131.349 \\
True Anomaly (deg) & 360.000 \\
\hline
\end{tabular}

about the surface of Mars and (2) analysing the types, contents, and spatial distribution of minerals on the surface of Mars.

MMS can obtain the visible and infrared images with high-resolution reflectance spectra of the key areas of Martian surface and can map the global surface using its multispectral mode. It has the optional ability to acquire continuous spectral sampling and interval sampling of the whole spectrum. In addition, it has the ability of in-flight calibration.

In the past 20 years, a large amount of Mars surface imaging spectral data collected by Visible and Infrared Mineralogical Mapping Spectrometer (OMEGA) onboard Mars Express and Compact Reconnaissance Imaging Spectrometer for Mars (CRISM) onboard Mars Reconnaissance Orbiter (MRO), has accumulated extremely high scientific contributions to Martian surface materials, polar deposits, atmosphere, and satellites of Mars. The OMEGA (Bonello et al. 2004; Bibring et al. 2005, 2006; Bakker et al. 2014) is integrated by a pushbroom imaging spectrometer consists of a visible channel push-broom spectrometer and an infrared, which together can map the Martian surface from $0.36-5.02 \mu \mathrm{m}$ with a spectral sampling of $7.5-23 \mathrm{~nm}$. The spatial resolution is about $0.3-4.8 \mathrm{~km} / \mathrm{pixel}$ in the mission orbit. CRISM (Murchie et al. 2007, 2009) focuses on high-resolution reconnaissance. Due to the along-track motion compensation, CRISM achieves a spatial resolution of up to $18 \mathrm{~m} /$ pixel at a $300-\mathrm{km}$ orbital altitude, and performs imaging spectral measurement from $0.36-3.92 \mu \mathrm{m}$ with a spectral sampling of $6.55 \mathrm{~nm} / \mathrm{channel}$. The spatial resolution of MMS is similar to that of OMEGA and inferior to CRISM, but the spectral resolution is better. This paper provides an overview of the design, operation, and performance of MMS and is divided into following major sections: (1) overview of MMS and instrument operations; (2) opto-mechanical, hardware, and software design of MMS instruments; (3) calibration and performance; (4) laboratory data that illustrate instrument performance; (5) summary and conclusions.

\section{Instrument Overview and Mission Analysis}

\subsection{Instrument Composition}

Tianwen-1's Mars orbiter and MMS are shown in Fig. 1a, and Fig. 1b. The parameters of remote sensing orbit are shown in Table 1. During times at an altitude of less than $800 \mathrm{~km}$, MMS will perform nadir observation according to the telemetry commands. Near the apogee, it will conduct in-flight calibration through orbiter attitude adjustment every month, which is described in Fig. 1c.

An overview of MMS is shown in Fig. 2. The instrument is comprised of two parts: (1) the main body and (2) the thermal control accessories (TCA), which are connected to the cold 


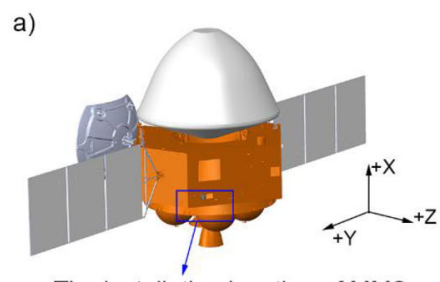

The installation location of MMS

b)

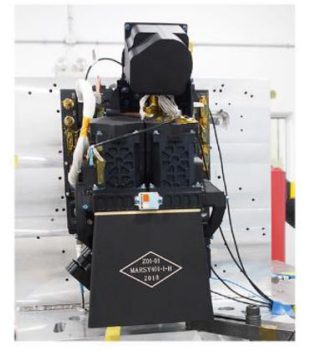

c) Nadir detection stage (orbit height below $800 \mathrm{~km}$ )

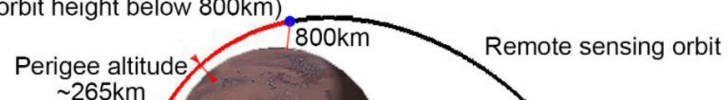

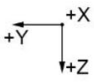

The top view of MMS in random vibration test. The body size is $350 \mathrm{~mm}(\mathrm{~L}) \times 198 \mathrm{~mm}(\mathrm{~W}) \times 244 \mathrm{~mm}(\mathrm{H})$.

Fig. 1 The orbiter and the remote sensing orbit. a) Installation location of MMS on Tianwen-1's Mars orbiter; b) The top view of MMS; c) Schedule of MMS in remote sensing orbit. The period of the elliptical orbit is 7.8 hours, and the duration of nadir observation stage is about 26 minutes

Optical Sensor Unit (OSU)

V-NIR module and N-MIR module

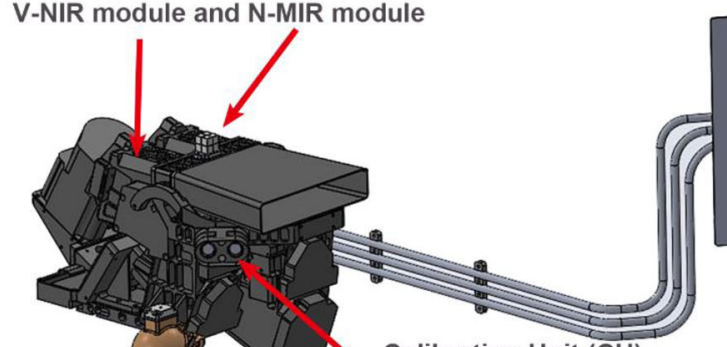

Thermal Control Accessories (TCA)

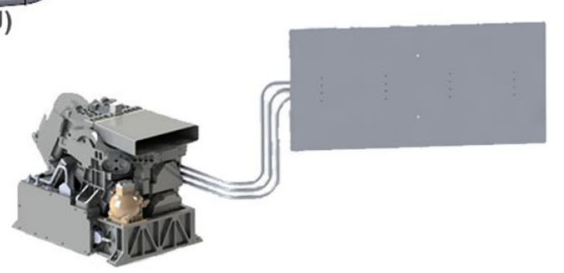

Fig. 2 System configuration diagram of MMS, including OSU, EU, CU, and TCA. The OSU and EU adopt separate designs, thermal accommodations. CU includes a solar viewer and an integrating sphere, each of which are connected by Y-type fibres. TCA's heat spreader is installed on the $+Y+Z$ panel of the orbiter, using heat pipes to cool the N-MIR spectrometer

plate of the orbiter to ensure a low-temperature state of MMS. The main body of MMS consists of the Optical Sensor Unit (OSU), Calibration Unit (CU), and Electronics Unit (EU). The OSU consists of the visible- and near-infrared (V-NIR) module and the near- and middle-infrared (N-MIR) module, both designed to operate separately. Both modules have fore-optics, slit, spectrometers, and calibration accessories linked to integrating sphere and 


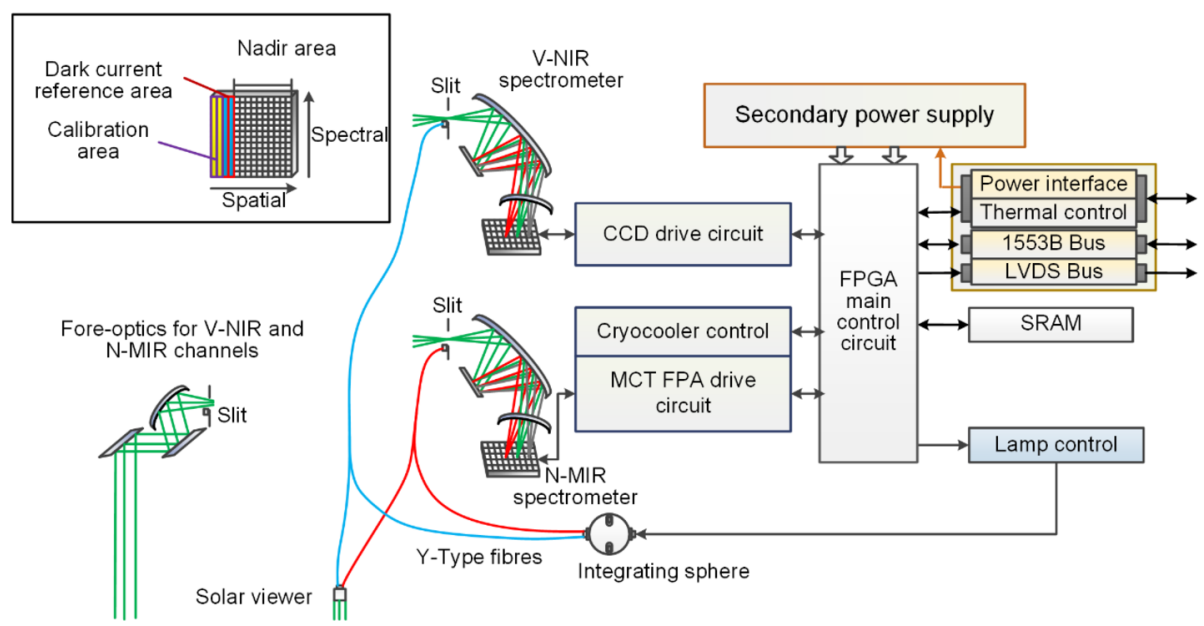

Fig. 3 Schematic diagram of the internal structure and working principle of MMS

cosine receiver of the solar viewer via fibre and micro-optic parts, which are together called as CU. The EU includes a power supply, master controller, charge-coupled device (CCD) driver, focal plane array (FPA) driver, telemetry control, and data transmission.

The instrument block diagram is shown in Fig. 3, and its features include: (1) the system has independent fore-optics and spectrometers (for miniaturization and for a compact optical path distribution, a free-form surface combined with a reflective grating has been adopted to realize a wide-spectrum and highly efficient spectral detection); (2) use of on-chip processing to achieve a variety of operation modes to adapt to the needs of different spatial resolutions and spectral ranges; (3) internal and external in-flight calibration; and (4) passive thermal control with a heat pipe and expansion plate in order to suppress background heat radiation.

\subsection{Specifications and Operation Modes}

MMS meets the scientific objective to analyse the types, abundance and spatial distribution of minerals on Martian surface, and its specifications are shown in Table 2.

The main system is divided into two channels, which differ in spectral range, sampling interval and spectral resolution. The V-NIR module of the OSU adopts a CCD array detector for acquisition of spectral images in the spectral range of $0.379-1.076 \mu \mathrm{m}$ with spectral sampling better than $3 \mathrm{~nm}$, whereas the N-MIR module adopts a mercury-cadmium-telluride focal plane array (MCT FPA) to obtain spectral information over the 1.033-3.425 $\mu \mathrm{m}$ range with spectral sampling better than $8 \mathrm{~nm}$.

The instantaneous field of view (IFOV) of the two channels is designed to both be 0.5 $\mathrm{mrad}$, and the effective full field of view (FOV) of nadir detection is $12^{\circ}$. The effective scales of the two detectors are equivalent to 512 columns $\times 255$ rows with $26-\mu$ m elements in the V-NIR channel and 512 columns $\times 320$ rows with $25-\mu$ m elements in the N-MIR channel, respectively. Out of these, samples $0-415$ are used for nadir observations and the rest are used for dark current reference acquisition and in-flight calibration.

Considering the enormous volume of data transmission bandwidth on the imaging spectrometer's scientific data downlink, several pixel binning schemes with real-time data processing were adopted for nadir observation, dominated by multi-sample hyperspectral mode 
Table 2 MMS Specifications

\begin{tabular}{|c|c|}
\hline Parameters & Measurement \\
\hline Spectral range & $\begin{array}{l}0.379-1.076 \mu \mathrm{m} \text { with } 2.73 \mathrm{~nm} / \mathrm{band} @ \mathrm{~V}-\mathrm{NIR} \\
1.033-3.425 \mu \mathrm{m} \text { with } 7.5 \mathrm{~nm} / \mathrm{b} \text { and @ N-MIR }\end{array}$ \\
\hline Spectral resolution & $\begin{array}{l}2.96-3.90 \mathrm{~nm} @ \mathrm{~V}-\mathrm{NIR} \\
8.36-10.91 \mathrm{~nm} @ \mathrm{~N}-\mathrm{MIR}\end{array}$ \\
\hline $\begin{array}{l}\text { Instantaneous field } \\
\text { of view }\end{array}$ & $0.50 \mathrm{mrad}$ \\
\hline True field of view & $12^{\circ}$ for nadir observation \\
\hline $\begin{array}{l}\text { Modular transfer } \\
\text { function }\end{array}$ & $\geq 0.21 @ 19.2$ lp/mm in V-NIR channel and $0.21 @ 20$ lp/mm in N-MIR channel \\
\hline Quantisation & 12 bits \\
\hline Signal-to-noise ratio & $\begin{array}{l}>45 @ \text { V-NIR }(405 @ 0.75 \mu \mathrm{m}),>33 @ \mathrm{~N}-\mathrm{MIR}(253 @ 2.3 \mu \mathrm{m}), \text { at } 1.52 \mathrm{AU}, \\
\text { phase angle of } 45^{\circ}, \text { and albedo of } 15 \%\end{array}$ \\
\hline Power consumption & Peak $44.9 \mathrm{~W}$ and average $29.1 \mathrm{~W} @$ optical temperature of $-15^{\circ} \mathrm{C}$ \\
\hline Mass & Body with multilayer insulation: $7.26 \mathrm{~kg}$ and thermal control accessories: $0.83 \mathrm{~kg}$ \\
\hline
\end{tabular}

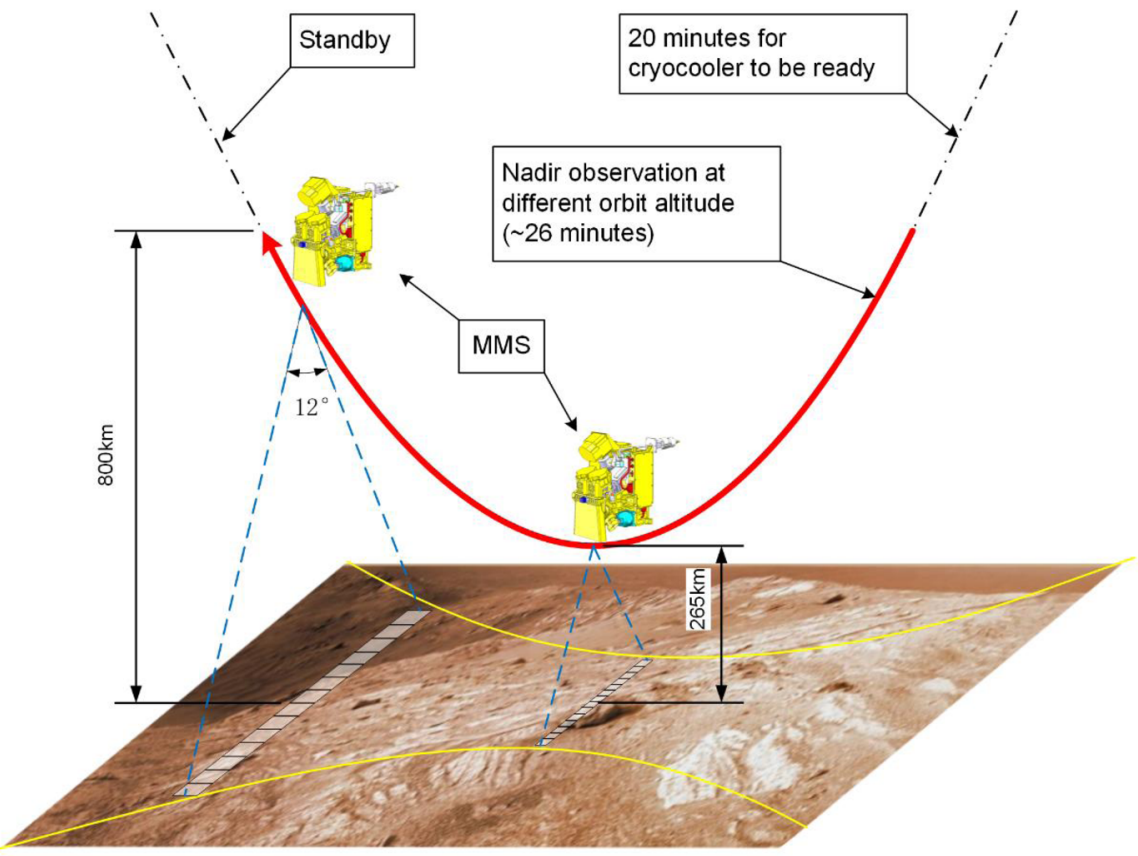

The Martian surface

Fig. 4 Schematic representation of MMS's nadir observation. The cryocooler needs to complete the cool the MCT FPA before reaching an orbital altitude of $800 \mathrm{~km}$, and MMS performs nadir observation for 26 minutes approximately

and multispectral imaging mode. According to the highly elliptical orbit designed for remote sensing, nadir detection will be conducted in a continuously varying scheme dependent on orbital altitude, as shown in Fig. 4. At periapsis, the orbital altitude will reach a 
minimum of $265 \mathrm{~km}$, an altitude of less than $800 \mathrm{~km}$, and the effective detection time per orbit will be approximately 26 minutes. Supported by its capability of $0.5 \mathrm{mrad}$ IFOV and 416 samples cell array for nadir observation, the swath width of MMS is $\sim 55 \mathrm{~km}$ at an altitude of $265 \mathrm{~km}$. With the orbiter pointed to nadir, MMS can perform multi-sample hyperspectral mode imaging at full spectral resolution $(0.379-1.076 \mu \mathrm{m}$ with $2.73 \mathrm{~nm} / \mathrm{band}$, $1.033-3.425 \mu \mathrm{m}$ with $7.5 \mathrm{~nm} / \mathrm{band}, 2.1 \mathrm{~km} /$ pixel at $265 \mathrm{~km}$ ). The multispectral mapping mode provides an additional observing option, a subset of 72 bands and binned to minimum pixel footprints of $265 \mathrm{~m} /$ pixel resolution. The nadir observation of MMS is described in Fig. 4.

The multi-sample hyperspectral modes and multispectral imaging modes of MMS are defined by how the 416 pixels covering the scene are spatially binned, which is detailed in Table 3.

The multispectral imaging mode has been designed in cooperation with Key Laboratory of Lunar and Deep Space Exploration of the Chinese Academy of Sciences. Six sets of multispectral selections have been integrated in MMS's software for detailed mineral exploration on the surface of Mars. The versatile multispectral band set can be used to detect all mineral groups but with slightly lower accuracy (Liu et al. 2018), whereas the remaining five sets can be used to detect phyllosilicate, sulfate, carbonate, hydrated salt, and Fe oxides and primary silicates with high accuracy.

The integrating sphere and solar viewer provide in-flight calibration capability to MMS. MMS's stability can be monitored at any time by using the main or backup calibration lamps installed in the integrating sphere. In-flight calibration will be performed near the apoapsis of the remote sensing orbit monthly. The solar irradiance on the solar viewer of MMS and the dark current images over full detector area will be acquired via orbiter attitude adjustment. The calibration modes are also described in Table 3.

MMS develops a preliminary set of recommendations including global mapping and region of interest observation. Global mapping will focus on the distribution characteristics and relative proportions of the main diagenetic minerals on Mars, and the discovery of new areas of interest. Considering the data transmission limit, MMS-D-208 or MMS-D104 mode with versatile band set will be selected for global coverage, and a typical mineral distribution map on the Martian surface with a higher spatial resolution will be obtained. Focusing on the pre-selected landing area of the Chinese rover, large impact crater, sediment area, and volcanic area, etc., multispectral imaging mode with suitable band set or hyperspectral mode will be performed for the fine mineral types, based on the reflectance characteristics of those areas (Liu et al. 2021).

\section{Instrument Design}

\subsection{Optical Sensor Unit (OSU)}

The optical sensor includes two channels, the V-NIR channel and the N-MIR channel. The two channels have a similar and individual configuration. The three-mirror-anastigmat foreoptics comprises a primary, a secondary, and a tertiary. The spectrometer consists of a collimator freeform mirror (F1), a grating, a reimaging freeform mirror (F2), and a corrector lens (Hou et al. 2015; Yuan et al. 2018). Ray trace of the two channels are shown in Fig. 5. Aperture stop is on the secondary or grating. Specifications of the two channels are listed in Table 4. 
Table 3 Operation modes of MMS

\begin{tabular}{ll}
\hline Detection & Operation mode \\
\hline $\begin{array}{l}\text { Nadir } \\
\text { observation }\end{array}$ & $\begin{array}{l}\text { 3-sample-hyperspectral mode } \\
\text { (MMS-D-3) }\end{array}$
\end{tabular}

Description

26-sample-hyperspectral mode (MMS-D-26)

208-sample-multispectral imaging mode (MMS-D-208)

104-sample-multispectral imaging mode (MMS-D-104)

52-sample-multispectral imaging mode (MMS-D-52)

Geometric calibration imaging mode (MMS-C-G)

Full-frame imaging mode (MMS-D-512)
Spatially: $16 \times$ cross-track pixels and $16 \times$ along-track pixels are binned to form a sample. In cross-track direction, there are 26 samples altogether. Three samples are selected from the 26 samples via instructions.

Spectrally: 576 bands ( 575 bands for spectral, 1 band for smear correction).

The data frame size after data preprocessing is 3 samples $\times$ 575 bands.

Spatially: $16 \times$ cross-track pixels and $16 \times$ along-track pixels are binned to form a sample. In cross-track direction, there are 26 samples altogether: 25 samples for targets, 1 sample for dark current. Spectrally: 576 bands ( 575 bands for spectral, 1 band for smear correction).

The data frame size after data preprocessing is 25 samples $\times 575$ bands.

Spatially: $2 \times$ cross-track pixels and $2 \times$ along-track pixels are binned to form a sample. In cross-track direction, there are 208 samples altogether: 207 samples for targets, 1 sample for dark current.

Spectrally: 72 bands (71 bands for spectral, 1 band for smear correction).

The data frame size after data preprocessing is 207 samples $\times 71$ bands.

Spatially: $4 \times$ cross-track pixels and $4 \times$ along-track pixels are binned to form a sample. In cross-track direction, there are 104 samples altogether: 103 samples for targets, 1 sample for dark current.

Spectrally: 72 bands ( 71 bands for spectral, 1 band for smear correction).

The data frame size after data preprocessing is 103 samples $\times 71$ bands.

Spatially: $8 \times$ cross-track pixels and $8 \times$ along-track pixels are binned to form a sample. In cross-track direction, there are 52 samples altogether: 51 samples for targets, 1 sample for dark current.

Spectrally: 72 bands (71 bands for spectral, 1 band for smear correction).

The data frame size after data preprocessing is 51 samples $\times 71$ bands.

Spatially: No pixels binned in cross-track and along-track, 512 samples (416 samples for targets, others for dark current and radiometric calibration).

Spectrally: 2 bands ( 1 band for V-NIR and the other band for N-MIR).

The data frame size after data preprocessing is 416 samples $\times 2$ bands.

Spatially: No pixels are binned in cross-track or along-track, 512 samples (416 samples for targets, others for dark current and radiometric calibration).

Spectrally: 576 bands ( 575 bands for spectral, 1 band for smear correction).

The data frame size after data preprocessing is 416 samples $\times 575$ bands. 
Table 3 (Continued)

\begin{tabular}{|c|c|c|}
\hline Detection & Operation mode & Description \\
\hline \multirow[t]{4}{*}{$\begin{array}{l}\text { In-flight } \\
\text { calibration }\end{array}$} & $\begin{array}{l}\text { Integrating sphere mode } \\
\text { (MMS-C-L) }\end{array}$ & $\begin{array}{l}\text { Spatially: } 1 \text { sample, average for every } 16 \text { acquisitions. } \\
\text { Spectrally: } 576 \text { bands ( } 575 \text { bands for spectral, } 1 \text { band for } \\
\text { smear correction). } \\
3 \text { groups of light turning on status, } 3 \text { group of turning off } \\
\text { status }\end{array}$ \\
\hline & $\begin{array}{l}\text { Solar observation mode } \\
\text { (MMS-C-S) }\end{array}$ & $\begin{array}{l}\text { Spatially: } 1 \text { sample, average for every } 16 \text { acquisitions. } \\
\text { Spectrally: } 576 \text { bands ( } 575 \text { bands for spectral, } 1 \text { band for } \\
\text { smear correction). }\end{array}$ \\
\hline & $\begin{array}{l}\text { Dark current reference } \\
\text { (MMS-DC) }\end{array}$ & $\begin{array}{l}\text { Spatially: } 1 \text { sample, average for every } 16 \text { acquisitions. } \\
\text { Spectrally: } 576 \text { bands ( } 575 \text { bands for spectral, } 1 \text { band for } \\
\text { smear correction). }\end{array}$ \\
\hline & $\begin{array}{l}\text { Space observation mode } \\
\text { (MMS-C-FD) }\end{array}$ & $\begin{array}{l}\text { Spatially: No pixels are binned in cross-track or along-track, } \\
512 \text { samples (for full frame dark background). } \\
\text { Spectrally: } 576 \text { bands ( } 575 \text { bands for spectral, } 1 \text { band for } \\
\text { smear correction). }\end{array}$ \\
\hline
\end{tabular}

a)

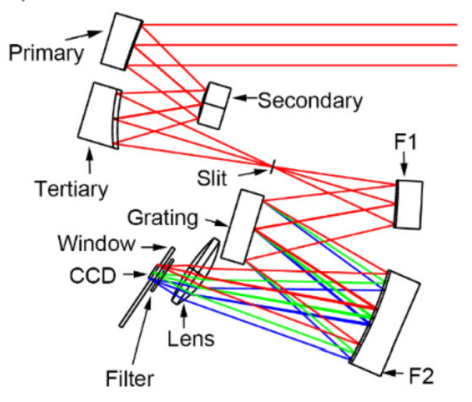

b)

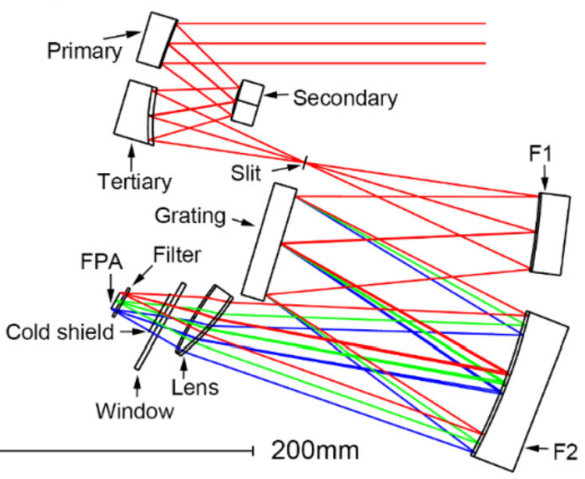

Fig. 5 Ray trace of MMS's optics: a) V-NIR channel; b) N-MIR channel. Each channel has its own optical system, including fore-optics and spectrometer. The two fore-optics are the same. The fore-optics and spectrometer of each channel were designed individually and then integrated together

All the mirrors are aluminium integrated into opto-mechanical designed (Yuan et al. 2017; Cook and Silny 2010). The OSU's configuration is shown in Fig. 6. The left shows the V-NIR channel, and the right shows the N-MIR channel.

The fore-optics are nearly telecentric in image space, providing a good pupil match with the spectrometer design and enough imaging space for the calibration module, which is set near the slit. The specifications and performance of the fore-optics are described in Table 5. The maximum distortion is $0.117 \mathrm{~mm}$ along the line field, which equals an FOV of $0.11^{\circ}$. The distortion was precisely measured via geometric calibration and could be corrected by image rectification (Mouroulis et al. 2007). The hood of the fore-optics is $125 \mathrm{~mm}$ long. The stray light coefficient of the fore-optics is less than $\mathrm{e}^{-4}$.

All the mirrors were fabricated using single-point diamond turning (SPDT). The assembly and alignment process is ensured by the high precise mechanical reference (Yuan et al. 2016). Apart from the assembly and optical surfaces, the mirrors, including the primary, 
Table 4 Specifications of the OSU

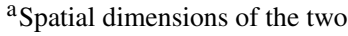
detectors are utilised for imaging as well as radiation calibration; 416 elements are for spatial imaging and the remaining 96 elements are for radiation calibration

Table 5 Specifications and performance of the fore-optics

\begin{tabular}{lll}
\hline Parameters & V-NIR & N-MIR \\
\hline Scan type & Push-broom & Push-broom \\
Spectral range & $0.4-1.1 \mu \mathrm{m}$ & $1.0-3.4 \mu \mathrm{m}$ \\
Spectral sampling & $2.73 \mathrm{~nm}$ & $7.50 \mathrm{~nm}$ \\
Bands & 256 & 320 \\
Working F/number & 2.96 & 2.84 \\
Cross-track FOV & $12^{\circ}$ & $12^{\circ}$ \\
IFOV & $0.5 \mathrm{mrad}$ & $0.5 \mathrm{mrad}$ \\
Slit width & $31 \mu \mathrm{m}$ & $31 \mu \mathrm{m}$ \\
Spectrometer magnification & $-0.845 \times$ & $-0.8125 \times$ \\
Detector array size & $512^{\mathrm{a}} \times 256$ (binned) & $512^{\mathrm{a}} \times 320$ \\
Detector pixel size & $26 \mu \mathrm{m}$ square (binned) & $25 \mu \mathrm{m}$ square \\
\hline
\end{tabular}

a)

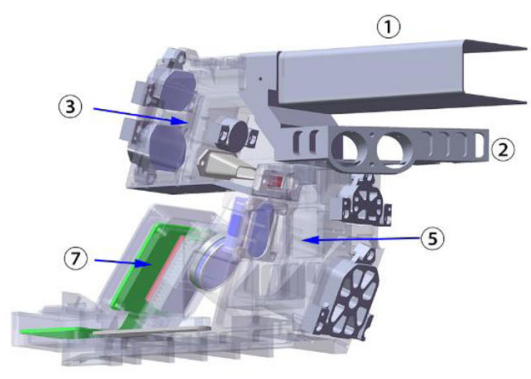

b)

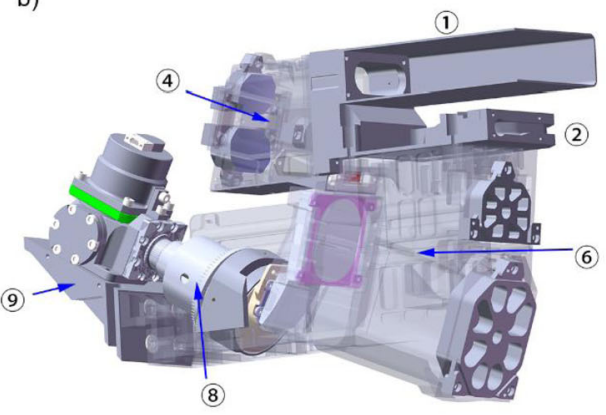
(1) Hood (2) Fore-optics holder
(3) V-NIR fore-optic
(4) N-MIR fore-optic
(5) V-NIR spectrometer
(6) N-MIR spectrometer (7) CCD
(8) FPA (9) Cryocooler holder

Fig. 6 OSU profile (sides and top). a) The opto-mechanical design of the V-NIR channel; b) The opto-mechanical design of the M-NIR channel. In the actual model, the two channels are arranged in parallel. Here, for the convenience of illustration, the two channels are shown in the left figure (V-NIR channel) and the right figure (M-NIR channel) respectively

secondary, and tertiary of the fore-optics are blackened. The optical surface is silver plus protective film coated. Taking the V-NIR fore-optics as an example, the root mean square (RMS) error of the wavefront as measured by the interferometer for the centre field of view is 0.094 wave; the deviations of the measured values from the design values for the other representative field of views are less than 0.02 , which is receivable according to the tolerance analysis, confirming good alignment. 
Table 6 Specifications and performance of the spectrometers

\begin{tabular}{lll}
\hline Parameters & V-NIR & N-MIR \\
\hline Spectral range & $0.4-1.1 \mu \mathrm{m}$ & $1.0-3.4 \mu \mathrm{m}$ \\
Spectrometer magnification & $-0.845 \times$ & $-0.8125 \times$ \\
Objective normal aperture & 0.143 & 0.143 \\
Slit scale & $12.8 \mathrm{~mm} \times 31 \mu \mathrm{m}$ & $12.8 \mathrm{~mm} \times 31 \mu \mathrm{m}$ \\
Groove lines & $150 \mathrm{~g} / \mathrm{mm}$ & $30 \mathrm{~g} / \mathrm{mm}$ \\
Smile (design/measure) & $4.26 \mu \mathrm{m} / 15.85 \mu \mathrm{m}$ & $4.52 \mu \mathrm{m} / 25.00 \mu \mathrm{m}$ \\
Keystone (design/measure) & $12.88 \mu \mathrm{m} / 13.26 \mu \mathrm{m}$ & $5.04 \mu \mathrm{m} / 5.75 \mu \mathrm{m}$ \\
\hline
\end{tabular}

a)

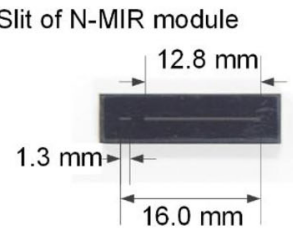

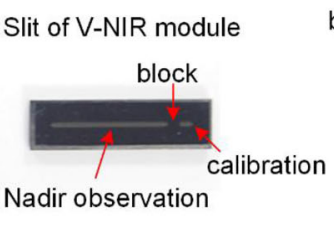

b)

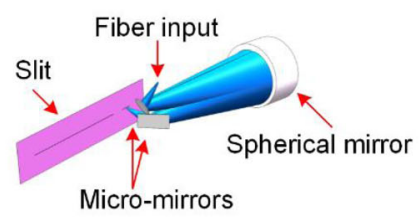

Fig. 7 The air slits applied to MMS. a) The illustration of the slits for the V-NIR and N-MIR channels. Lengths of the nadir area, block area and calibration area are $12.8 \mathrm{~mm}, 1.9 \mathrm{~mm}$ and $1.3 \mathrm{~mm}$ respectively. The block areas are used for the dark current reference acquired. b) The micro-optical parts assembled on the side of the V-NIR slit for coupling fiber-optic signals. The micro-optical part of the N-MIR channel has the same structure as the one of the V-NIR channel

Spectrometers of the two channels have a similar structure but different parameters. The optical structure of the spectrometer is semi-reflective with a flat grating and freeform optics. A single freeform mirror F1 is utilised as the collimator, a freeform mirror F2 together with an adhesive lens as the reimager, and a flat reflective grating as the dispersion element. Both the collimator and reimager mirrors were designed as extended polynomial surface type. The adhesive off-axis lens near the imaging place has been utilised to enhance the relative aperture and improve image tilt for detector placement. The specifications and performance of the spectrometers are described in Table 6.

The air slits applied to MMS are divided into three areas: the nadir slit area, the block area, and the calibration area, and symmetrical in two spectrometers, as shown in Fig. 7. The calibration source from the fibres is focused on the calibration area on the slit, and the space target is separated by the block area on the slit with calibration source without confliction, which were imaged on the detectors. Both the slits have 512 elements along the spatial dimension, where 416 elements are used for imaging and 48 elements are used for calibration, and the residual 48 elements are used for separation. The field of views of the two bands were measured to co-register; for example, the 205th spatial pixel of the V-NIR corresponds to the 208th spatial pixel of the N-MIR, whereas the 0th spatial pixel of the V-NIR corresponds to the 413th spatial pixel the N-MIR.

The assembly of the spectrometers is similar to that of the fore-optics. After the separate assembly of each element or component to the main frame, alignment of the spectrometer refers to the clocking of the grating and the precise positioning of the CCD or the FPA. An auxiliary two-dimensional slit has been used (Yuan et al. 2019), as shown in Fig. 8. After the precise assembly of the grating and the detector, the auxiliary two-dimensional slit is replaced with the formal slit.

Smile and keystone of the imaging spectrometers of the two bands were measured precisely during calibration. Keystone is less than 0.13 pixel in V-NIR channel and less than 


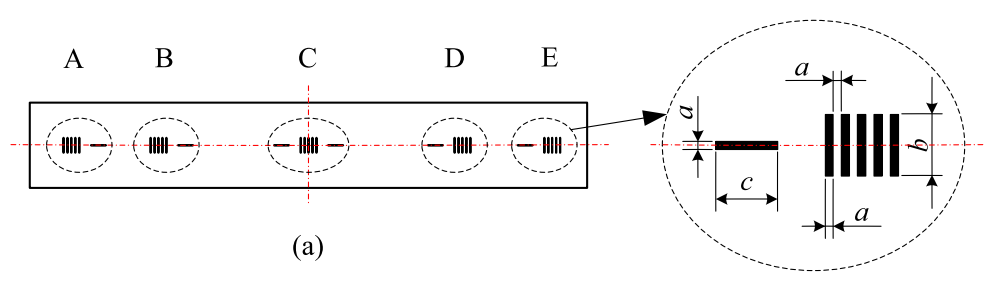

Fig. 8 Sketch map of the auxiliary two-dimensional slit. A to E stand for field points. Here, $a$ equals to one pixel size, $b$ and $c$ are given appropriate values as needed. The difference between the auxiliary two-dimensional slit and the formal slit is that auxiliary slit has sub-slits along the spatial and spectral dimensions. In that case, the spectral response of IFOV and the spatial response of monochromatic spectrum can be monitored by auxiliary slit, for quick-adjusting of gratings and detectors

Table 7 Distribution characteristics of system MTF on image plane

\begin{tabular}{llll}
\hline & Spatial pixel 30th & Spatial pixel 208th & Spatial pixel 400th \\
\hline Band 230 of V-NIR & 0.24 & 0.23 & 0.26 \\
Band 128 of V-NIR & 0.43 & 0.37 & 0.30 \\
Band 50 of V-NIR & 0.34 & 0.34 & 0.21 \\
Band 214 of N-MIR & 0.21 & 0.29 & 0.25 \\
Band 160 of N-MIR & 0.25 & 0.32 & 0.33 \\
Band 14 of N-MIR & 0.23 & 0.32 & 0.27 \\
\hline
\end{tabular}

0.23 pixel in N-MIR channel. Smile is less than 0.66 pixel in V-NIR channel and less than 1.00 pixel in N-MIR. The modulation transfer function responses of representative FOV points at the band 128 of the V-NIR channel and at the band 160 of the N-NIR channel are shown in Fig. 9, and the distribution characteristics are shown in Table 7. The average MTF of the spectrometers of the V-NIR and N-MIR bands are better than $0.21 @ 19.21 \mathrm{p} / \mathrm{mm}$ and $0.21 @ 20 \mathrm{lp} / \mathrm{mm}$, respectively.

The detector in the V-NIR module is a CCD47-20 Back Illuminated NIMO FrameTransfer CCD by Teledyne e2v (Teledyne 2017), with high quantum efficiency and low readout noise. Its spectral coverage is $200-1050 \mathrm{~nm}$. The total image section of this CCD is $1024 \times 1024$ with $13 \mu \mathrm{m}$ elements. The highest reading rate can reach $5 \mathrm{MHz}$, and it is capable of dual-port simultaneous reading. The detailed information of the CCD is shown in Table 8. The middle region of the image section is used for dispersion imaging (scale is $1024 \times 510$ ), as shown in Fig. 10a, whereas the other regions were abandoned. The dark reference rows on the top of the image section are read for smear correction. Then the CCD is readout via $2 \times 2$ pixel binning, making the final image scale as $512 \times 256$, as shown in Fig. 10b; and the long axis oriented in the along-slit direction. The CCD and the readout circuit are jointly mounted on the detector bracket of the V-NIR module. The detector is covered with a sapphire protection window that is plated with a dark stripe matching the slit block area, which is used for obtaining the dark current reference spectra synchronously during in-orbit operation. Finally, an order-sorting filter (OSF) has been installed on the surface of the protection window to block the higher-order diffraction rays from the grating.

The FPA (Boltar et al. 1999) used in the N-MIR module of OSU was developed by Shanghai Institute of Technical Physics (SITP), and consists of an MCT FPA packaged in a dewar, an OSF, and a Stirling cryocooler, which provides a temperature control capacity of $80 \mathrm{~K}$ (operation) or $150 \mathrm{~K}$ (long-standby). The Stirling cryocooler has been screened by 

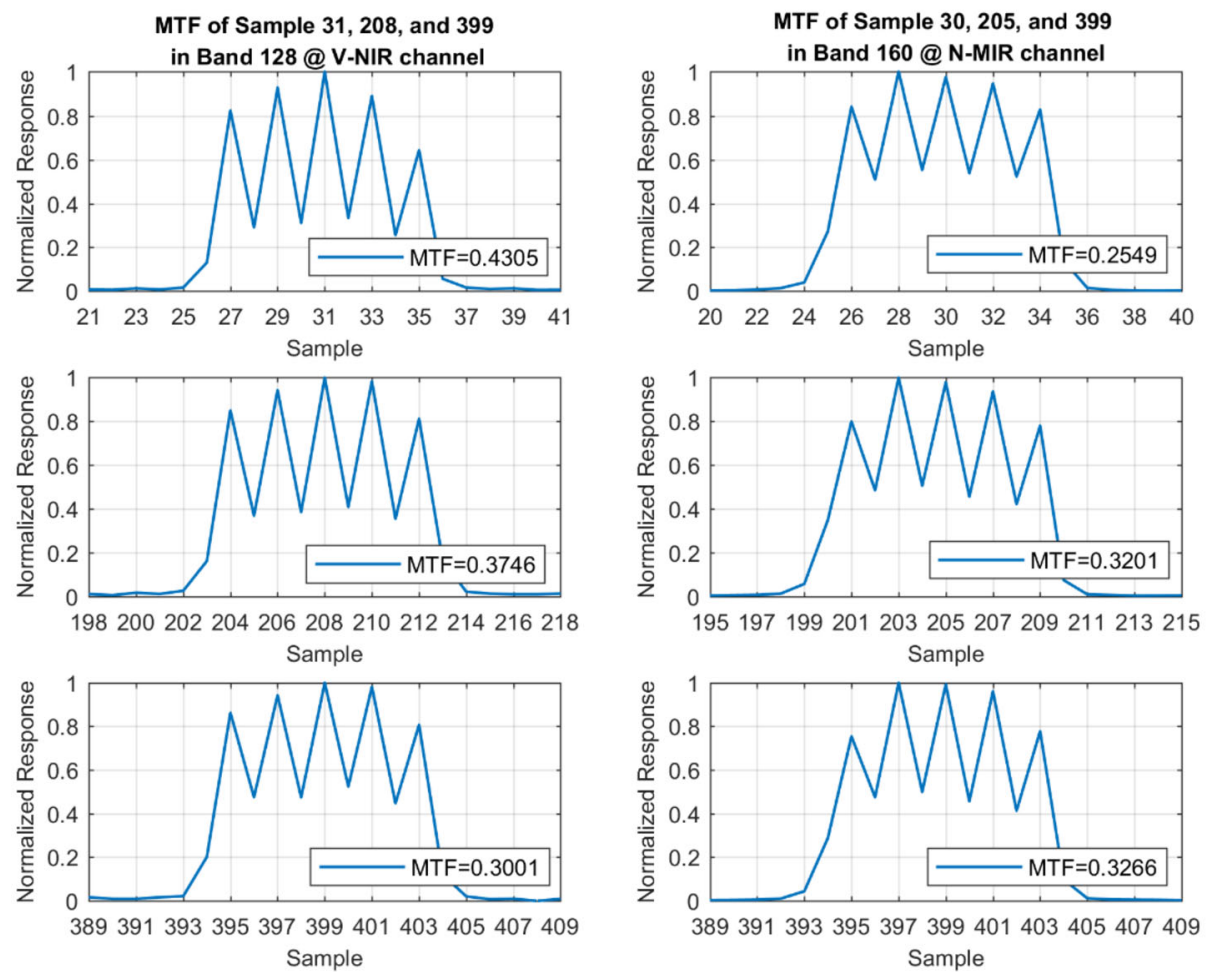

Fig. 9 The MTF test results for Band 128 of V-NIR channel and Band 160 of N-MIR channel

aerospace tests, and the input power consumption of that is $9.19 \mathrm{~W}$ under operation mode. The MCT FPA consists of 512 columns $\times 320$ rows with $25 \mu \mathrm{m} \mathrm{HgCdTe}$ elements, and the long axis oriented in the along-slit direction, as shown in Fig. 10c. A $19 \mathrm{~mm} \times 21.8 \mathrm{~mm}$ cold baffle, plated with black nickel inside and polished outside, is mounted on the top of the FPA surface to mask the thermal background from outside the FOV. The blind pixel rate of the flight model FPA is $0.55 \%$. When the temperature of the target blackbody is $300 \mathrm{~K}$, the signal-to-noise ratio (S/N) of the FPA in half-potential well at $80 \mathrm{~K}$ operation temperature is 223.8 with a 12-bit AD. In this case, the average noise of the detector is 3.05 , equivalent to $1.525 \mathrm{mV}$. The specification of the FPA is shown in Table 8 .

The main stray light in the spectrometer comes from the other-order diffraction rays of the grating. General stray lights, such as multiple reflections (of the filter, the window of the detector, and the other optical surfaces) and scattering, are suppressed by a coating, by using appropriate mechanical design, and by blackening. The other-order diffraction rays of the grating of each spectrometer are decreased using an OSF placed in close proximity to the FPA. The OSF of the V-NIR channel is a general filter with two coating segments, and the boundary design is $550 \mathrm{~nm}$, which is shown in Fig. 11a. The cut-off depth of 200-350 nm is less than $1 \%$, and the average transmittance of $400-800 \mathrm{~nm}$ is better than $95 \%$ in the Filter A, while the cut-off depth of $200-550 \mathrm{~nm}$ is less than $1 \%$, and the average transmittance of $600-1100 \mathrm{~nm}$ is better than $95 \%$ in the Filter B. However, one of the N-MIR channels is slightly different as it has three coating segments. The transmitted wavelengths of the three zones are 1.0-1.9 $\mu \mathrm{m}, 1.4-2.7 \mu \mathrm{m}$, and 2.1-3.4 $\mu \mathrm{m}$, respectively, as shown in Fig. 11b. The cut-off depth is less than $3 \%$, and the average transmittance is better than $92 \%$ in the N- 

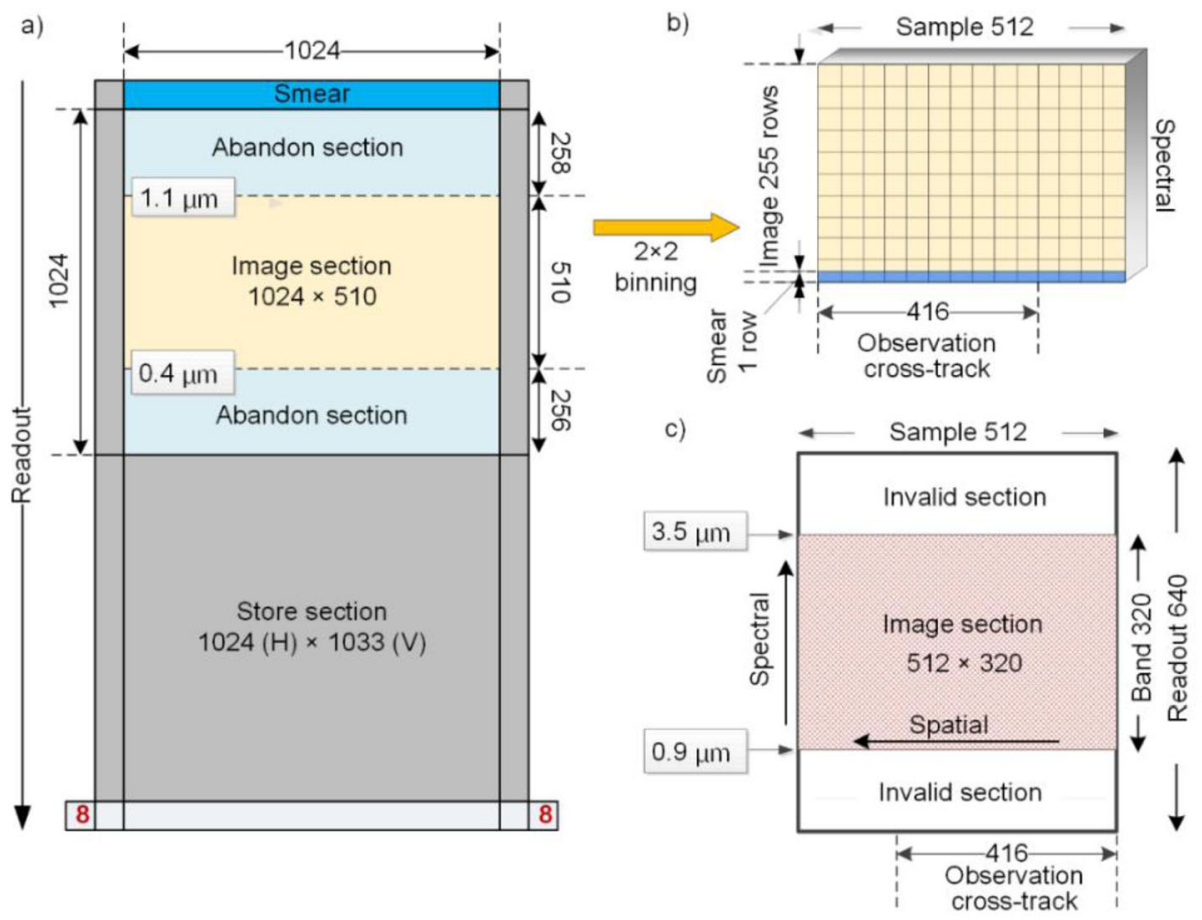

Fig. 10 The CCD readout and output format in operation mode of MMS-D-512. a) The top view of CCD structure, and only the middle 510 rows of image section are used for spectral imaging and the others are abandoned. b) After $2 \times 2$ binning, the readout image resolution of the CCD is $512 \times 256$ with a smear reference at the end. c) The scheme of FPA readout

Table 8 Key specifications of detectors on MMS

\begin{tabular}{|c|c|c|c|}
\hline \multicolumn{2}{|l|}{$\mathrm{CCD}$} & \multicolumn{2}{|l|}{ MCT FPA } \\
\hline Parameters & Typical value & Parameters & Typical value \\
\hline Active pixels & $1024 \times 1024$ & Array size & $512 \times 320$ \\
\hline Pixel size & $13 \times 13 \mu \mathrm{m}$ & Pixel size & $25 \mu \mathrm{m} \times 25 \mu \mathrm{m}$ \\
\hline Storage pixels & $1024 \times 1033$ & Temperature range & $80 / 150$ K Controllable \\
\hline Peak output voltage & $540 \mathrm{mV}$ & Output saturation voltage & $2.225 \mathrm{~V}$ \\
\hline Dynamic range & $50,000: 1$ & Frame frequency & $60.44 \mathrm{~Hz}$ \\
\hline Number of output amplifiers & 2 & Temperature stability & $0.2 \mathrm{~K} @ 30 \mathrm{~min}$ \\
\hline Output amplifier responsivity & $4.5 \mu \mathrm{V} / \mathrm{e}^{-}$ & Number of GPIO & 4 bus \\
\hline Readout noise at $253 \mathrm{~K}$ & $2 \mathrm{rms} \mathrm{e}^{-} /$pixel & Temperature control precision & $-0.3 \mathrm{~K}$ \\
\hline Maximum readout frequency & $5 \mathrm{MHz}$ & Non-linearity & 0.00764 \\
\hline $\begin{array}{l}\text { Dark signal non-uniformity } \\
\text { at } 293 \mathrm{~K}\end{array}$ & $2 \mathrm{ke}^{-} /$pixel & Non-uniformity & $4.80 \%$ \\
\hline Weight & $7.5 \mathrm{~g}$ & Weight & $765.7 \mathrm{~g}$ \\
\hline Package size & $22.7 \times 42.0 \mathrm{~mm}$ & Integration capacitance & $1.6 \mathrm{Me}^{-}$ \\
\hline
\end{tabular}



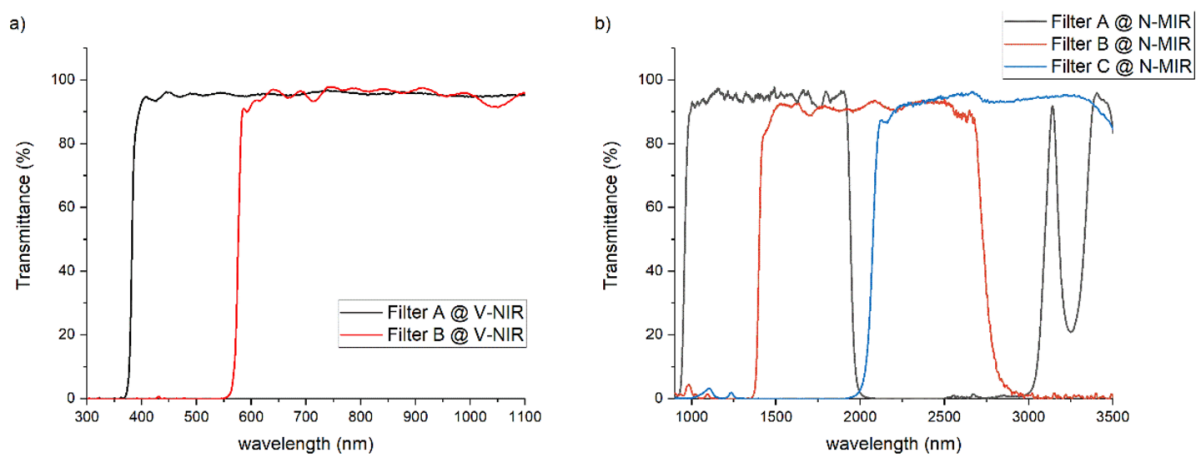

Fig. 11 Optical efficiency measurements of V-NIR and N-MIR OSFs. a) The transmittance of the OSF used in V-NIR channel, the boundary of two coating segments is designed as $550 \mathrm{~nm}$. b) The transmittance of the OSF used in N-MIR channel, the transmitted wavelengths of the three zones are 1.0-1.9 $\mu \mathrm{m}, 1.4-2.7 \mu \mathrm{m}$, and 2.1-3.4 $\mu \mathrm{m}$, respectively

MIR channel's OSF. The filter weakens the second- and even higher-order stray diffraction rays effectively. But as for segment $\mathrm{A}$, the coating cannot cut off clearly after the $3.2 \mu \mathrm{m}$ wavelength, thus infrared radiation background cannot be ignored, which is dealt using the calibration and algorithm. The stray light coefficient of the fore-optics is less than $\mathrm{e}^{-3}$. Due to the implementation of these stray light suppression measures, the stray light suppression coefficient of the spectrometer is less than $\mathrm{e}^{-3}$ as well.

\subsection{Calibration Unit (CU)}

In order to calibrate MMS in orbit, a light source with known spectral characteristics should be used as the calibration source to compare the spectral response characteristics with the pre-launch state of the instrument and to provide reference data for the processing and application of in-orbit detection data. Considering feasibility, weight, and reliability requirements, the in-flight $\mathrm{CU}$ includes internal and external calibration sources, as shown in Fig. 12. For internal calibration, the integrating sphere is equipped with lamps (Murchie et al. 2007) whose exit light is coupled to the spectrometer slit through fibres in order to monitor the stability of the system in different stages. For external calibration, the solar viewer, as a cosine receiver, receives solar irradiance and introduces it into the spectrometers' slits via fibres' to track the source near the apogee of the remote sensing orbit. MMS has two detection modules, and each needs a Y-type fibre to transmit the calibration source from solar viewer and integrating sphere. The fibre core diameter is $105 \mu \mathrm{m}$, the normal aperture (NA) is 0.22 , and fibre materials used in the two modules are quartz (for V-NIR) and ZBLAN (for N-MIR). On the slit side of the fibre, the cores are arranged in the direction of the slit and the micro-optics are designed to couple calibration lights into the slits.

The internal calibration scheme uses a gold-plated integrating sphere with two calibration lamps, as shown in Fig. 12. The lamps are located on the top and bottom of the sphere, and the exit port is located in the middle. Two baffles are arranged to prevent the light from reaching the exit port directly. On the exit port, light in the integrating sphere is coupled into the fibre fixed via a SubMiniature version A (SMA) port using miniature mirrors. The solar viewer has been integrated into the system, maintaining a $45^{\circ}$ angle with the nadir observation direction. The surface of the solar viewer is a sapphire scattering plate, designed as a cosine receiver. The effective receiving surface is $11 \mathrm{~mm}$ in diameter and $2 \mathrm{~mm}$ in thickness. 


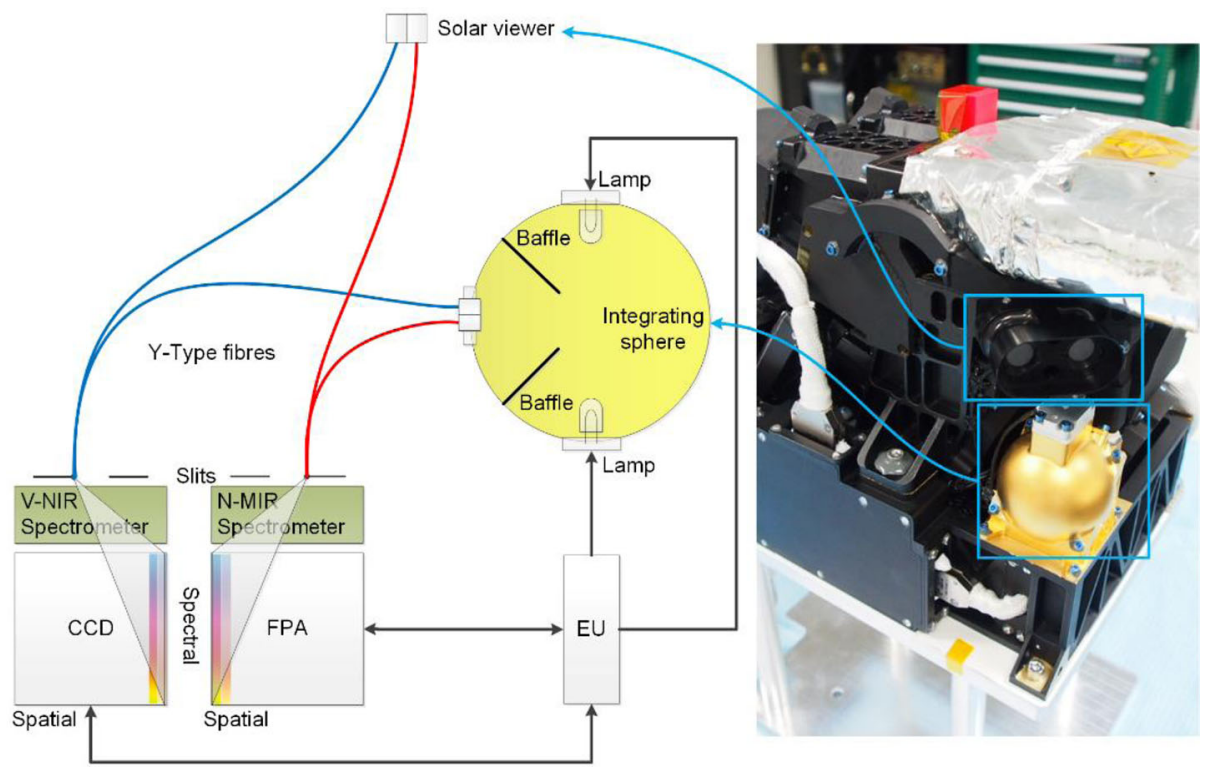

Fig. 12 Design of on-orbit calibration scheme for MMS. The integrating sphere of MMS for in-flight calibration

\subsection{Electronics Unit (EU)}

The EU mainly consists of six modules: main control module, V-NIR module, N-MIR module, lamp control and telemetry module, power converter module, and external interface. In addition to the field programmable gate array (FPGA), external programmable read-only memory (PROM), and electrically erasable PROM (EEPROM), the main control module also contains the 1553B engineering parameter acquisition circuit, a low-voltage differential signalling (LVDS) high-speed digital transmission circuit, and a static random-access memory (SRAM) storage control circuit. The V-NIR module consists of a CCD area array detector and its bias, driver, and readout circuits, whereas the N-MIR module consists of the FPA with cryocooler and its bias, driver, readout, and cooling control circuits. The lamp control and telemetry module include the on/off control circuits for the two built-in lamps and the instrument's internal temperature and voltage telemetry circuits. The external interface module includes a primary power interface, a remote telemetry interface, a 1553B bus interface, an LVDS high-speed data interface, and a thermal power interface. The power converter module receives $29 \mathrm{~V}$ primary power provided by a load controller, and uses DC/DC conversion circuits to generate secondary power at $+30 \mathrm{~V},+24 \mathrm{~V}, \pm 12 \mathrm{~V}$, and $+5 \mathrm{~V}$. The other voltages required for the equipment are generated by low-dropout regulators (LDOs). The overall block diagram and the flight model (FM) of MMS's EU are shown in Fig. 13 and Fig. 14, respectively.

MMS uses an FPGA to control the Stirling cryocooler, CCD, and FPA. As the FPA needs to work at a low temperature (approximately $80 \mathrm{~K}$ ), it needs to be refrigerated before detection or calibration in-orbit. In addition, in order to reduce the switching times and stand-by consumption of the Stirling cryocooler, the control temperature of the cryocooler will be kept at $150 \mathrm{~K}$ by command when MMS is far away from the perigee of remote sensing orbit. The FPGA control of CCD and FPA, two detectors of MMS, mainly involves 


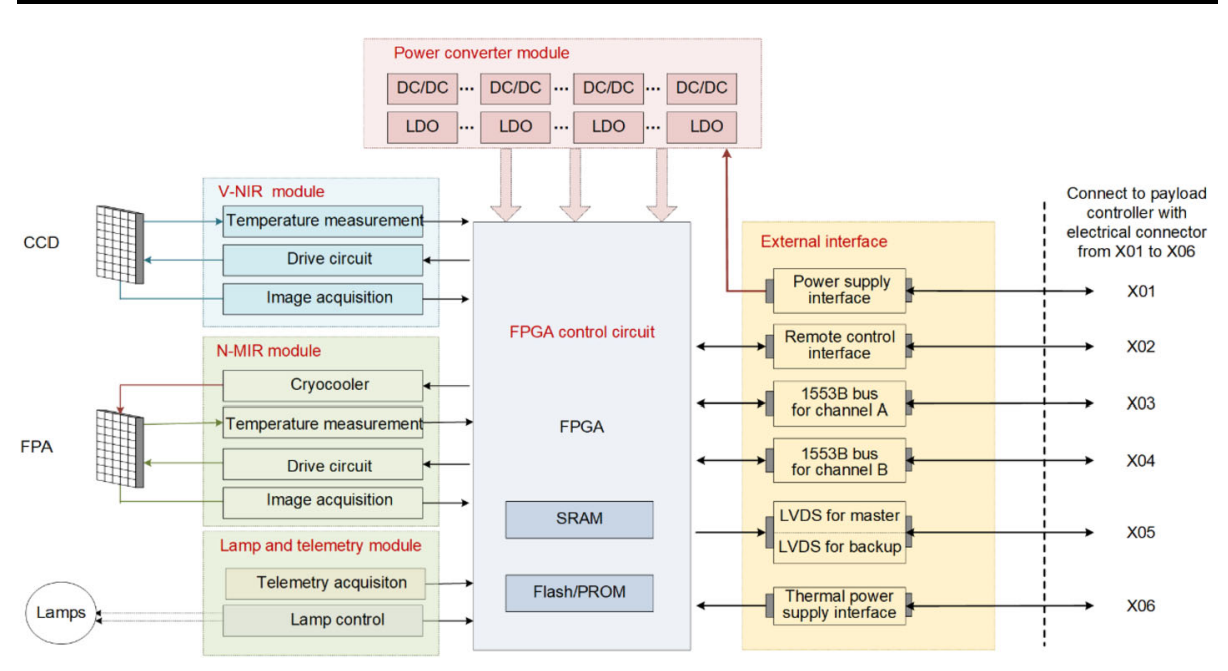

Fig. 13 Block diagram of the Electronics Unit
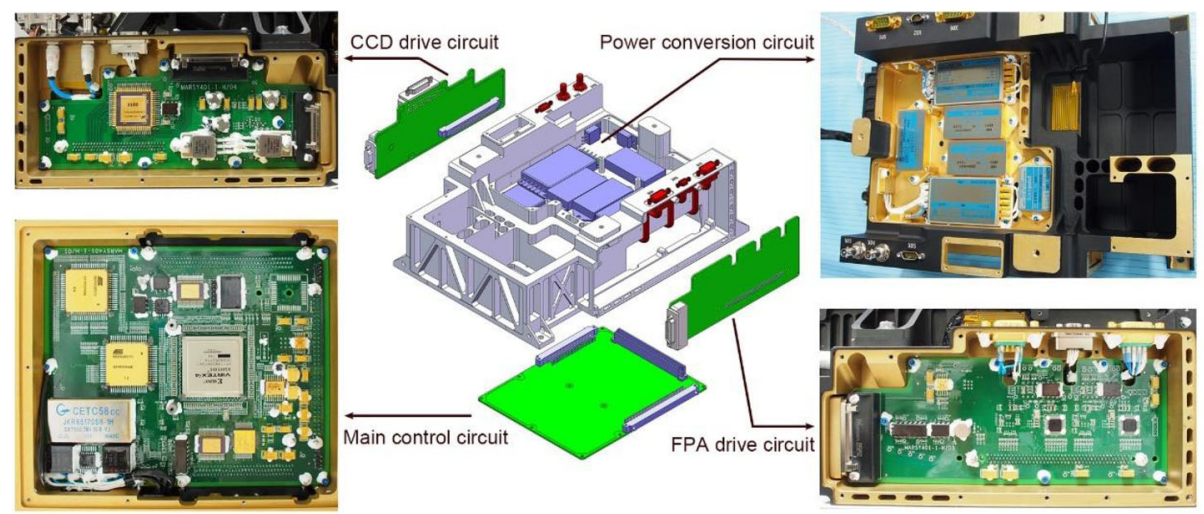

Fig. 14 Circuit distribution of EU. The power conversion module is integrated with the electronics box to facilitate heat dissipation. The main control circuit is installed from the bottom and connected to the power conversion module. The CCD drive circuit and the FPA drive circuit are placed on both sides of the main control circuit

frame rate and integration time. The sampling frame frequencies are $20 \mathrm{~Hz}$ and $32 \mathrm{~Hz}$ in different operation modes. The FPA exposure time is 1.536-23.04 ms, and the CCD uses two sets of exposure time, to cope with operation modes and the target under different lighting conditions. Based on data synchronization requirements, FPA acquisition is triggered by the CCD frame synchronization signal. The stable spectra of stationary light after lighting the lamp for $90 \mathrm{~s}$ in $\mathrm{CU}$ and the corresponding dark current after turning off were obtained for self-stability monitoring regularly. According to the short instruction received by the 1553B channel, MMS can realise a variety of in-orbit detection and calibration modes.

\subsection{Software Control}

The detector readout data is preprocessed on the chip, including blind pixel compensation, pixel binning, multi-spectral wavelength selecting, data combination, and compression. 


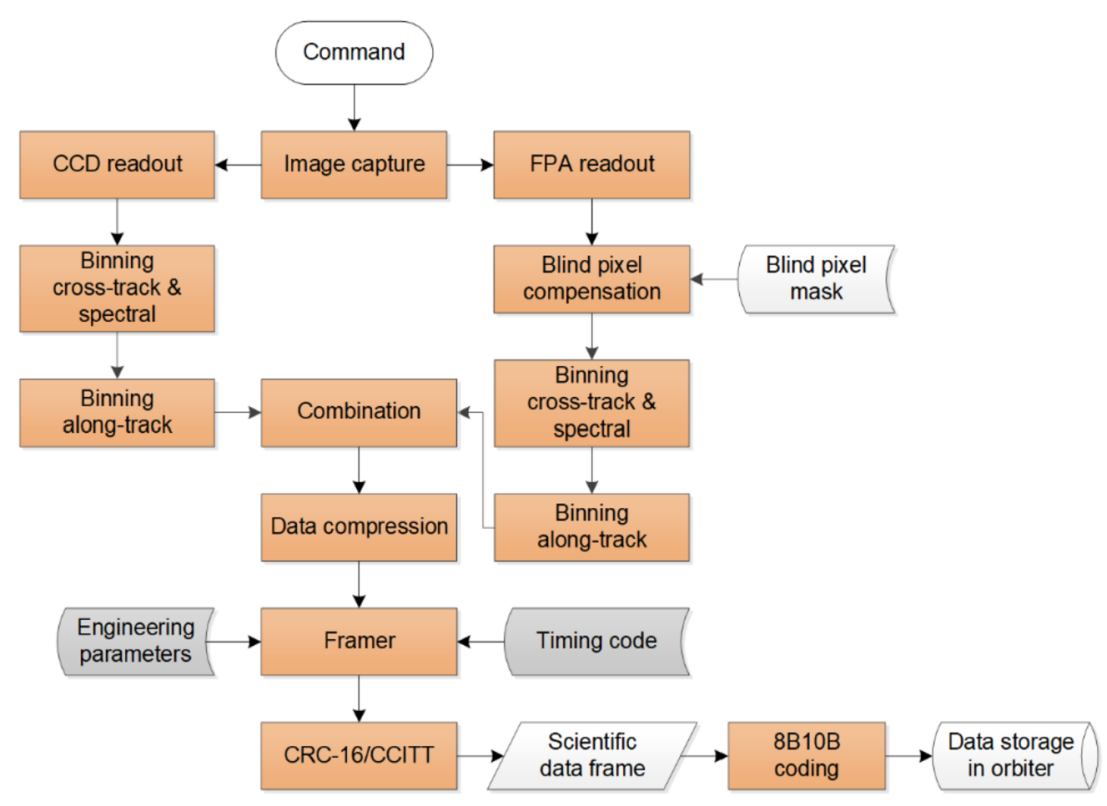

Fig. 15 Information flow on chip of MMS

Then, engineering telemetry, time code, and cyclic redundancy checks are added to form data frames, which are transmitted to the orbiter for centralised management after $8 \mathrm{~B} / 10 \mathrm{~B}$ coding. The information flow of the on-chip is shown in Fig. 15.

The location of blind pixels is stored in the EEPROM on MMS's EU and can be updated online. It adopts 1-bit quantisation to realize information storage of $512 \times 320$ blind pixel mask image. In parallel processing, only the actual pixel corresponding to the effective value of the mask image is operated by replacing it with the mean of 8 adjacent pixels $(3 \times 3)$. If the adjacent pixels also contain the pixel corresponding to the mask that has a valid value, the pixel is skipped and the remaining adjacent pixels are used. The mask is extracted from the target of cold background at $250 \mathrm{~K}$ environment temperature.

The imaging resolutions of the CCD and FPA detectors are $512 \times 256$ and $512 \times 320$, respectively. A readout frame rate of $20 \mathrm{~Hz}$ or $32 \mathrm{~Hz}$ will produce a huge amount of hyperspectral data, adding enormous pressure on data transmission. Through pixel binning and multi-spectral selection, MMS reduces the rate of in-flight data generation for more effective in-orbit detection, as shown in Fig. 16. According to the previous description, the imaging area of the detectors can be divided into 416 samples of nadir detection area, 48 samples of dark current area, and 48 samples of calibration function area. In MMS, band selection and pixel binning preprocessing are performed differently on the detectors' readout frame under the multi-sample hyperspectral mode and multispectral imaging mode.

In the multi-sample hyperspectral mode, MMS's detectors are exposed globally and push-broom the surface of Mars at a rate of $20 \mathrm{~Hz}$. After completing the blind pixel compensation on chip, 26-sample hyperspectral data is generated by the $16 \times$ cross-track and along-track pixels binning of the nadir detection region, meanwhile the dark current reference spectral data captured at the same time replace one of the samples for the purpose of data preprocessing. The 3-sample hyperspectral mode further reduces the amount of data, which is chosen from the 26-sample hyperspectral mode through instruction setting. 


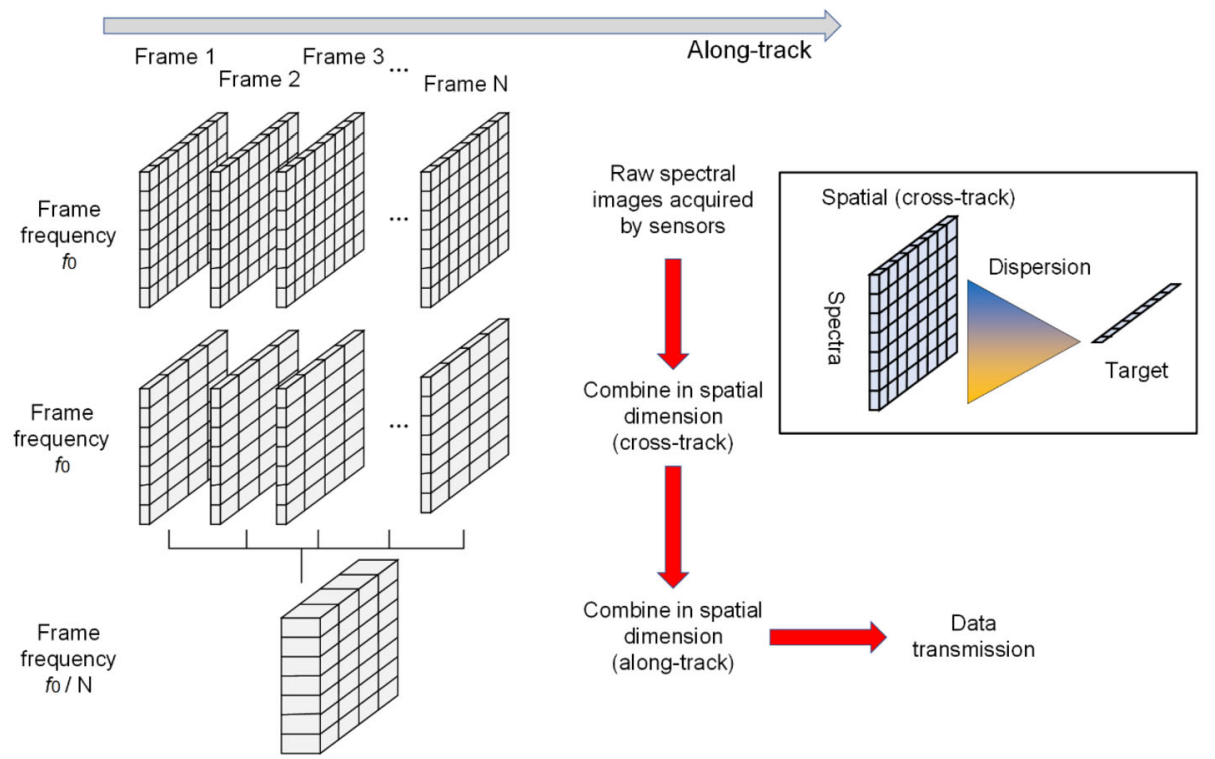

Fig. 16 Schematic diagram of pixel binning principle. The raw spectral images are binned cross-track firstly. Then pixel binning along-track is performed as shown. The spatial and spectral resolution differs according to the operation mode after pixel binning preprocessing

Compared to the multi-sample hyperspectral mode, which sacrifices spatial resolution, the multispectral imaging mode aims to obtain spectral information at a high spatial resolution. In this mode, a frame rate of readout data at $32 \mathrm{~Hz}$ is necessary according to the velocity-height ratio $(\mathrm{V} / \mathrm{H})$ of the orbiter in remote sensing elliptical orbit. Six band combination sets are preset in the ROM of FPGA to identify different mineral groups. All the combination sets have 72 bands, consisting of 71 bands for spectral coverage and an extra one for smear correction of the CCD detector. The $2 \times, 4 \times$, or $8 \times$ cross-track and alongtrack pixel binning is processed under command uplink.

The integration time of the FPA can be adjusted from 1.536-23.04 ms at steps of $1.536 \mathrm{~ms}$, which is suitable for the multi-sample hyperspectral and multispectral imaging modes. In contrast, the integration time of CCD has only two options. The minimum level is $5.155 \mathrm{~ms}$, and the other level is $44.635 \mathrm{~ms}$ in the multi-sample hyperspectral mode, and $25.68 \mathrm{~ms}$ in multispectral imaging modes, respectively. The readout frame rate, data frame rate, and optional integration time of MMS are detailed in Table 9.

Data compression is critical for storage and transmission of mass data processing in the field of deep space exploration due to the limited communication bandwidth available. Improved lossless JPEG (JPEG-LS, ISO-14495-1/ITU-T.87) performs excellently for lossless/near lossless compression, and it has become the preferred algorithm for image compression in space communications. The JPEG-LS compression algorithm for MMS was implemented by the K. Liu team at Xidian University (Belyaev et al. 2015). According to the data formats generated by various detection and calibration modes of MMS, lossless compression or near-lossless compression (compression ratio $>2: 1$ ) has been used for on-chip compression by adjusting the NEAR value, which means the maximum absolute error can be controlled in the encoder. 
Table 9 Valid integration times and frame rates of MMS

\begin{tabular}{lllll}
\hline Function & Integration time & Operation mode & $\begin{array}{l}\text { Frame rate } \\
\text { of readout }\end{array}$ & $\begin{array}{l}\text { Frame rate of } \\
\text { transmission after binned }\end{array}$ \\
\hline $\begin{array}{l}\text { Multi-sample } \\
\text { hyperspectral } \\
\text { mode }\end{array}$ & $\begin{array}{l}\text { CCD: } \\
5.155 \mathrm{~ms} / 44.635 \mathrm{~ms}\end{array}$ & $\begin{array}{l}\text { MMS-D-3 } \\
\text { MMS-D-26 }\end{array}$ & $\begin{array}{l}20 \mathrm{~Hz} \\
20 \mathrm{~Hz}\end{array}$ & $1.25 \mathrm{~Hz}$ \\
& FPA: & MMS-D-512 & $2 \mathrm{~Hz}$ & $2 \mathrm{~Hz}$ \\
& $1.536-23.04 \mathrm{~ms}$ & MMS-C-L & $20 \mathrm{~Hz}$ & $1.25 \mathrm{~Hz}$ \\
& & MMS-C-S & $20 \mathrm{~Hz}$ & $1.25 \mathrm{~Hz}$ \\
$\begin{array}{l}\text { Multispectral } \\
\text { imaging mode }\end{array}$ & CCD: & MMS-D-208 & $32 \mathrm{~Hz}$ & $16 \mathrm{~Hz}$ \\
& FPA: & MMS-D-104 & $32 \mathrm{~Hz}$ & $8 \mathrm{~Hz}$ \\
& $1.536-23.04 \mathrm{~ms}$ & MMS-D-52 & $32 \mathrm{~Hz}$ & $4 \mathrm{~Hz}$ \\
& & MMS-C-G & $32 \mathrm{~Hz}$ & $32 \mathrm{~Hz}$ \\
\hline
\end{tabular}

A unified data format has been used for transmission of scientific data in all operation modes, and the payload controller conducts transparent framing of MMS's scientific data.

\subsection{Thermal Control Accessories (TCA)}

To enhance the dynamic range through infrared background radiation suppression in-orbit, MMS adopts a passive thermal design to reduce the temperature of the OSU. The CCD detector is thermally mounted on the structural frame for heat dissipation, while the FPA adopts Stirling cryocooler to control the operation temperature of the sensor chip. When the orbital altitude is below $800 \mathrm{~km}$, FPA works at $80 \mathrm{~K}$. Otherwise, the temperature of the FPA is controlled at $150 \mathrm{~K}$ or shut down. The OSU and the integrating sphere are integrated above the EU by thermal installation design, forming a single instrument thermal zone. The main material used in the OSU is black anodised 6061T651 aluminium alloy, whereas the main material in the shell of the EU is MB8 magnesium alloy with thermal control black paint, both two surface treatments were used for heat dissipation. Two radiating surfaces have been used as well: one is located directly under the bottom plate and the other is located outside the inclined side plate of the orbiter. Both have been sprayed with white thermal control paint. The instrument has been installed on the bottom plate of the orbiter by thermal installation, and the contact surface is filled with thermal silicon grease. The heat dissipation loop is connected with 3 heat pipes with a diameter of $6 \mathrm{~mm}$ between the OSU and the radiation surface of the inclined side plate. An actual photo depicting the thermal control design is shown in Fig. 17a.

During the Earth-Mars transfer, capture, and parking stages, MMS will be kept at a low temperature. In order to survive, multiple thermal zones have been incorporated into the design. To reduce heat leakage, the hood and OSU are heat-insulated, and the multilayer insulation (MLI) is coated outside the hood (extra black film was wrapped in the FM) first. Then, a temperature compensation heater is set with a total power of $10 \mathrm{~W}$, of which $8 \mathrm{~W}$ are for OSU and $2 \mathrm{~W}$ are for EU. The heating circuit is controlled by a temperature relay with a closing temperature of $233 \pm 3 \mathrm{~K}$ and disconnection temperature of $247 \pm 3 \mathrm{~K}$. Throughout the vacuum heat balance test of the orbiter's FM, MMS storage temperature is around $238 \mathrm{~K}$. At the remote sensing stage shown in Fig. 17b, the operating temperatures of the OSU and EU are both lower than $250 \mathrm{~K}$. 

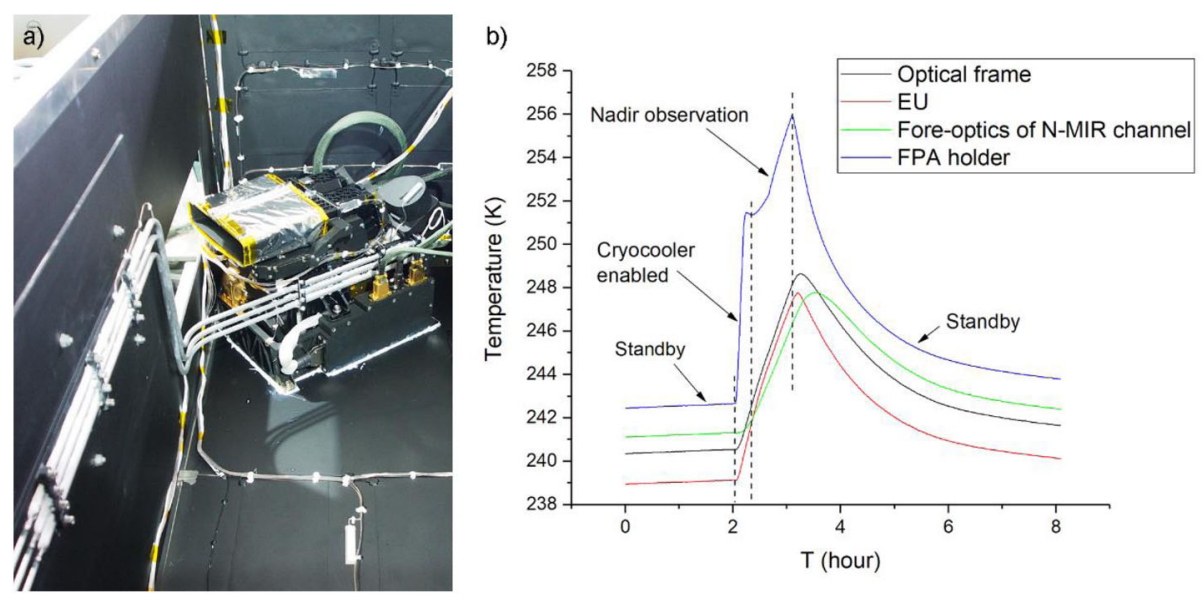

Fig. 17 a) The thermal control setting of MMS during the heat balance test of the EQM in the simulation orbiter platform. MMS installed on the bottom plate of the orbiter by thermal installation, and three extra heat pipes connected to the radiation surface of the orbiter. b) Temperature of MMS in vacuum heat balance test with the orbiter in FM stage. The optical frame and N-MIR channel's fore-optics heat up with the activation of cryocooler. The temperature rises from $242 \mathrm{~K}$ to $248 \mathrm{~K}$ during the 26 minutes period of nadir observation, and slowly returns to storage status as the cryocooler switch off or turn to low-power mode

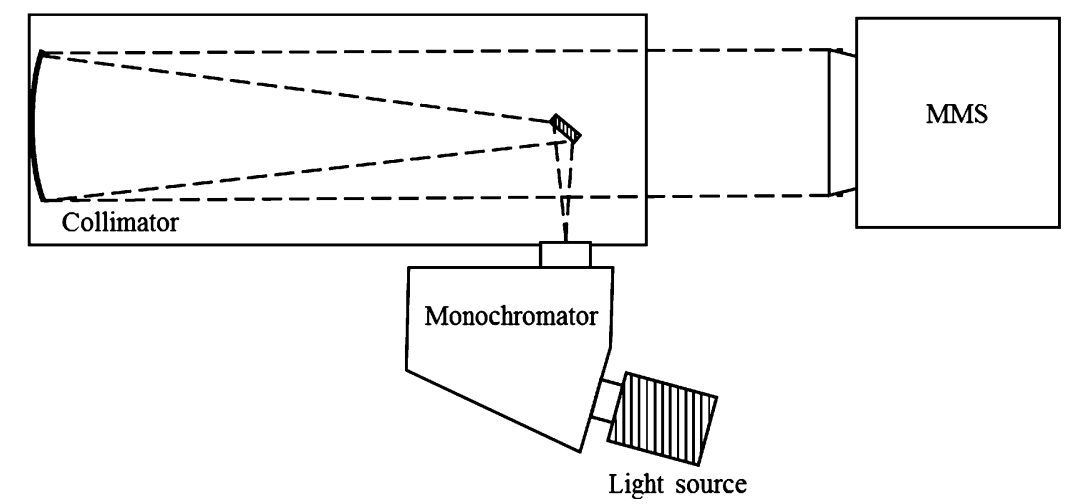

Fig. 18 Spectral calibration setup scheme

\section{Instrument Calibration}

\subsection{Pre-flight Calibration}

The imaging spectrometer requires spectral, radiometric, and geometric calibration preflight in order to convert the measured quantized intensity value into spectral radiance data products (Xu et al. 2012, 2014).

Figure 18 shows the setup scheme for the spectral calibration experiments, which consists of a light source, a monochromator, and a collimator. The purpose of the spectral calibration is to measure the spectral response function of MMS. The monochromator exit slit is located on the focal plane of the collimator for the monochromatic collimation beam. MMS 

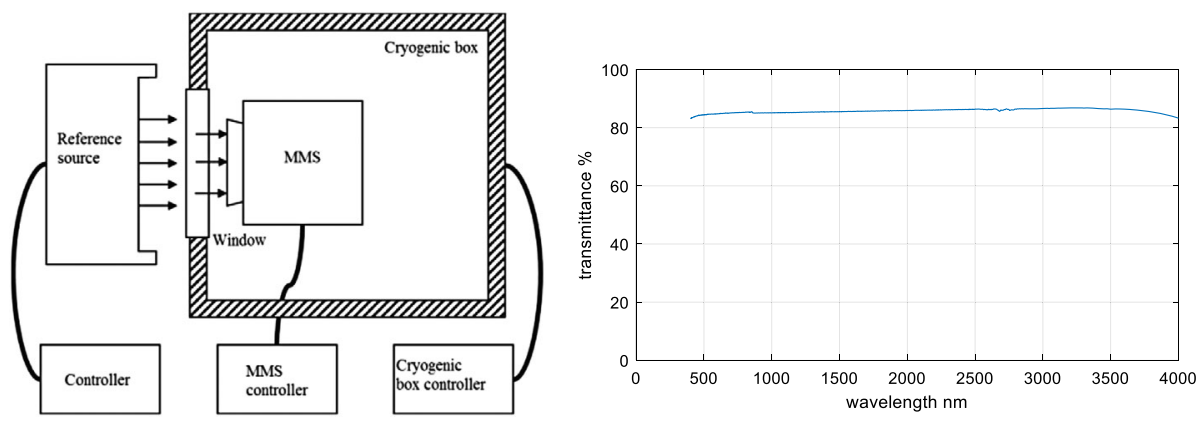

Fig. 19 Radiometric calibration setup and the transmittance of the sapphire window

Table 10 Temperature settings of cryogenic box for different experiments

\section{Experiments}

Temperature effect on dark background

Sensitivity non-uniformity

Temperature effect on sensitivity of nadir detection area

Absolutely radiometric calibration
Temperature

$238 \mathrm{~K}-268 \mathrm{~K}$

$253 \mathrm{~K}$

$238 \mathrm{~K}-268 \mathrm{~K}$ with an interval of $5 \mathrm{~K}$ $253 \mathrm{~K}$

was placed on the 2-dimensional rotation stage, which was used for switching between different FOVs through the azimuth axis as well as for eliminating fore-optics distortion effects through the pitch axis. The beam is received by MMS, and monochromatic spot radiation is formed on the detector arrays. Through the spectral scanning via the monochromator, the spectral response functions of different bands throughout the FOV were obtained.

Figure 19 shows the setup for radiometric calibration. The radiometric calibration system primarily consists of a reference source, a cryogenic box, and their controllers. MMS was placed in a cryogenic box to simulate the temperature of the operating environment in orbit, inhibit background radiation, and suppress the effects of gas absorption such as water vapour through nitrogen filling. Table 10 shows the temperature setup of the cryogenic box for different test experiments. The reference sources include an integrating sphere (LabSphere U200) and an area blackbody model (CES200-06), shown in Fig. 20. The integrating sphere has four halogen lamps, where the spectral radiance of Lamp No. 3 (L3 for short) can be adjusted by using a step shutter. The uniform surface light source with different radiance energy levels are generated through the lamp combination of the integrating sphere from visible to shortwave infrared, and the black body with different temperatures in the mid-infrared spectrum. The purpose of the radiometric calibration is to obtain the dark background estimation coefficients, the non-uniformity correction coefficients and the absolute radiometric coefficients.

The main purpose of geometric calibration is to determine MMS's internal orientation elements, including principal point position, principal distance, IFOV, and fore-optics distortion. The test setup scheme (Fig. 21) is similar to spectral calibration, primarily consisting of a point light source, a collimator, and a 2-dimensional rotation stage.

\subsubsection{Spectral Calibration}

In order to measure the spectral characteristics of different mineral targets, the spectral range, sampling step, and resolution of each band in MMS should be calibrated. In spec- 

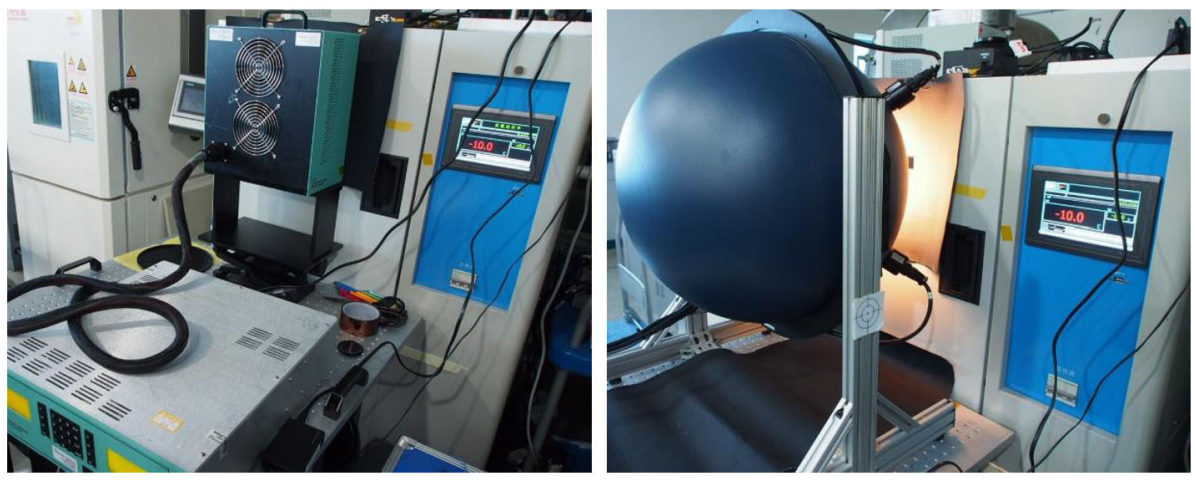

Fig. 20 Radiometric calibration at low temperature

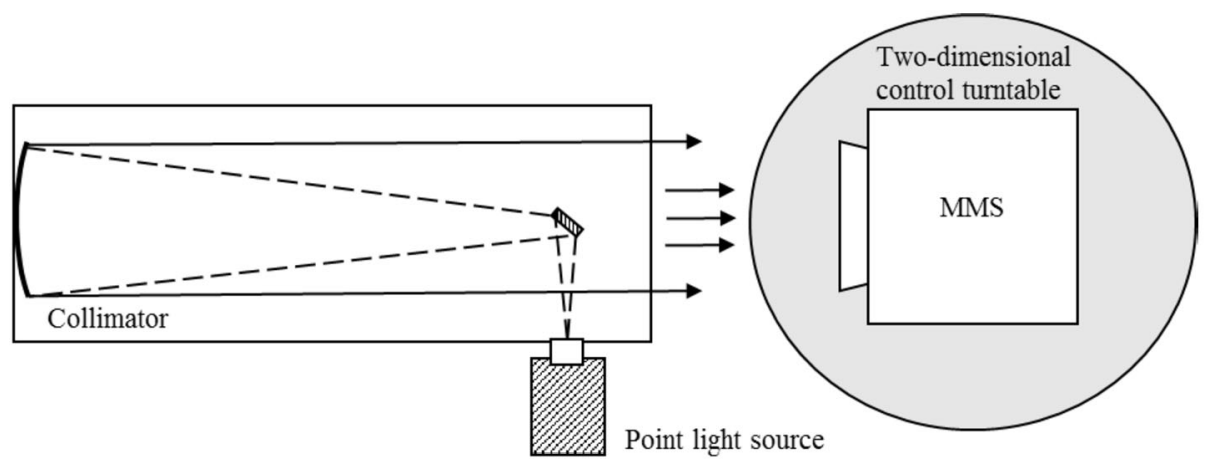

Fig. 21 Geometric calibration setup scheme

tral calibration, the exit slit of the monochromator was set to ensure that the dispersion bandwidth of the output beam was less than 1/10th of MMS's design full width at half maximum (FWHM). The centre wavelength and spectral resolution corresponding to the band of detectors were analysed via Gaussian curve fitting, as shown in Fig. 22.

In addition, by testing the polychromatic parallel light illumination conditions, the spectral keystone distribution of different samples on detectors were recorded, as shown in Fig. 23. Using the monochromatic parallel light signal, the spectral smile distribution characteristics on the detector are shown in Fig. 24.

\subsubsection{Dark Background}

Figure 25 shows the dark background images for V-NIR and N-MIR regions under an ambient temperature of $253 \mathrm{~K}$ different integration times in the V-NIR region. The integration time of V-NIR and N-MIR is $44.635 \mathrm{~ms}$ and $23.04 \mathrm{~ms}$, respectively. In the spatial dimension, the dark background in the V-NIR region is affected by two readouts, and the left side is approximately $10 \mathrm{DN}$ values higher than the right side. However, the dark background of N-MIR presents a striped feature in the spectral dimension, first decreasing and then increasing, and its profile curve is similar to the bowl curve. This effect is caused by the OSF in FPA, which has transmission characteristics in the mid-infrared $(>3.0 \mu \mathrm{m})$ in both $\mathrm{A}$ 

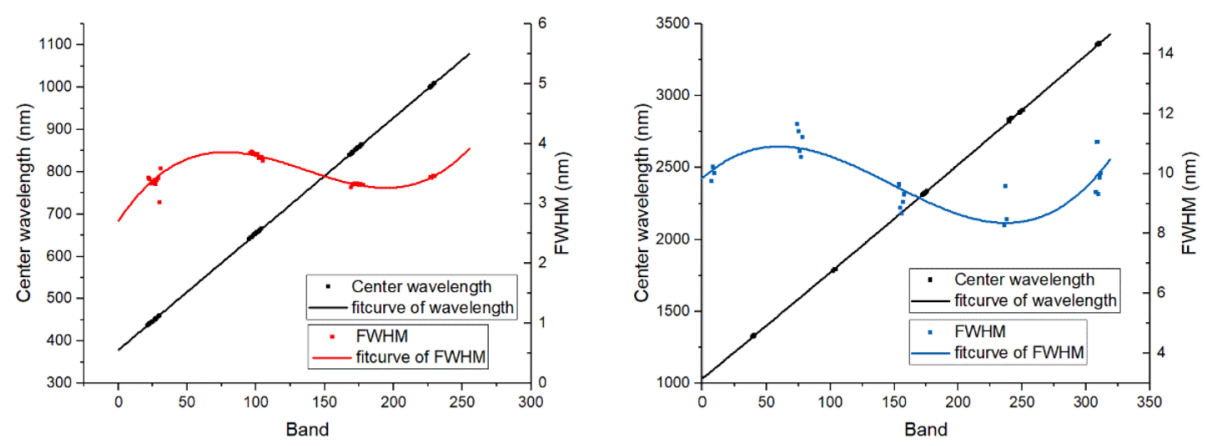

Fig. 22 V-NIR and N-MIR channels' spectral characteristics (centre wavelength and spectral resolution)
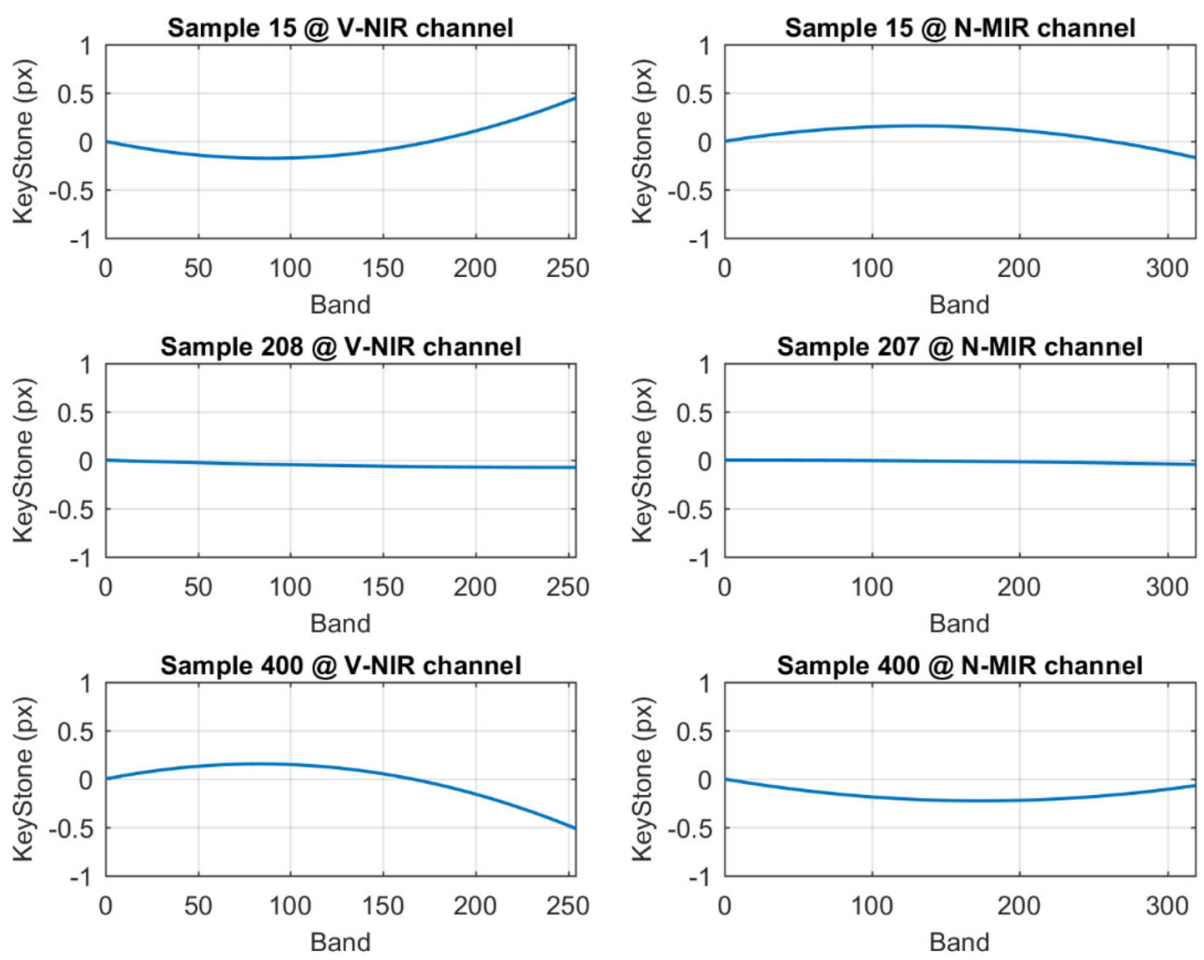

Fig. 23 Spectral keystone distribution characteristics of MMS; test results in samples 15, 208, and 400 of V-NIR channel and samples 15, 207, and 400 of N-MIR channel

and $\mathrm{C}$ zones (Fig. 11). Therefore, dark background in N-MIR region shows three stages, as shown in Fig. 25b.

MMS uses a part of the pixels for dark background monitoring (as shown in Fig. 3). In the ground test, we simulated the possible working environment temperatures of MMS with a low-temperature box, and obtained the relationship between the DN value of the pixel in the dark background monitoring and the imaging area without the input radiation. For the V-NIR region, we used the positive scale function to model the relationship. For the N-MIR 
a)
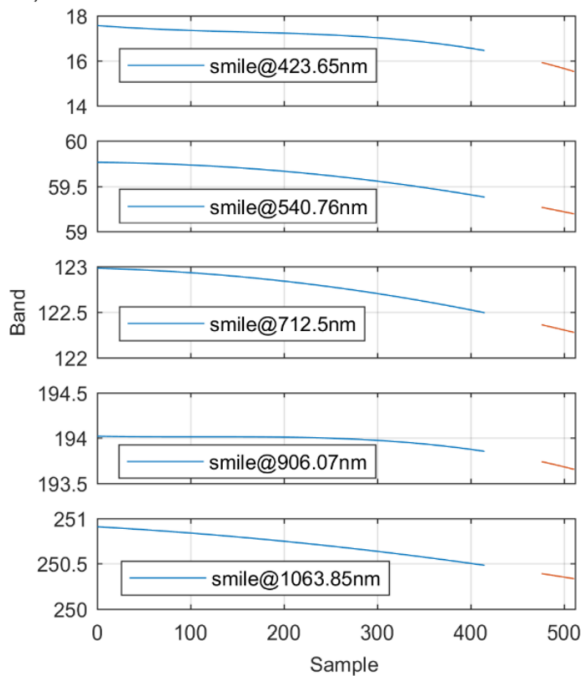

b)
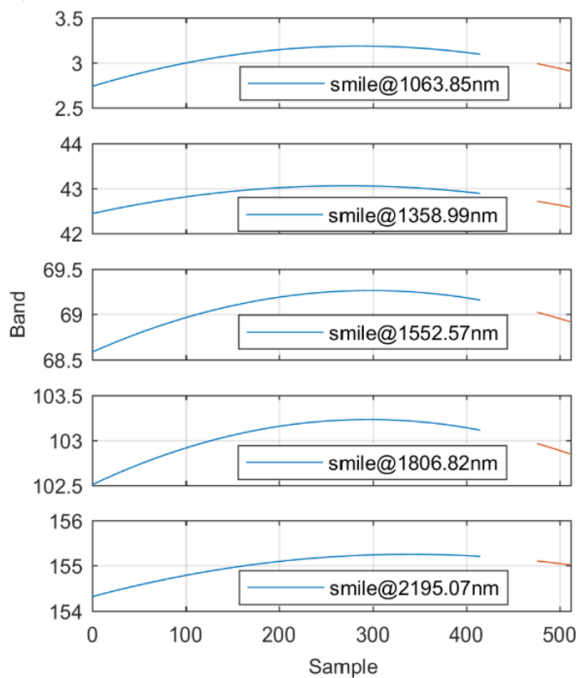

Fig. 24 Spectral smile distribution characteristics of MMS: a) V-NIR channel, b) N-MIR channel

a)

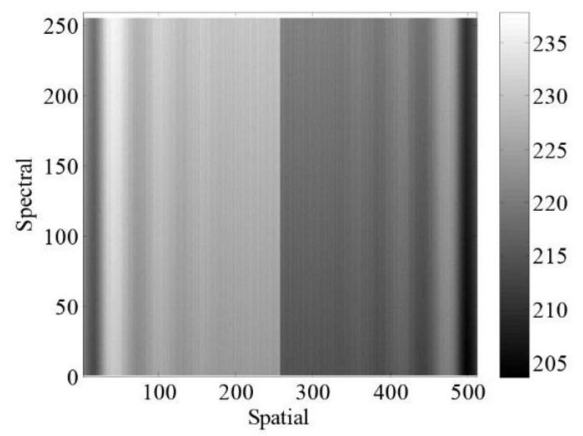

b)

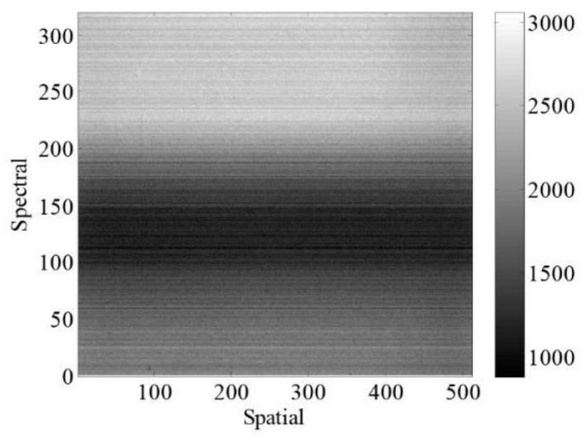

Fig. 25 Dark background images. a) V-NIR region with integration time of $44.635 \mathrm{~ms}$; b) N-MIR region with integration time of $23.04 \mathrm{~ms}$

region, the linear model was used. For in-flight operation, the DN value of each pixel in the imaging area can be estimated based on the DN value of dark background monitoring area and the models. As for the establishment of the test experiment and model, we will make a detailed introduction in another paper on MMS data processing and products.

\subsubsection{Smear Correction}

The detector of the V-NIR module is a frame-transfer CCD. The readout mechanism that the exposure process uses during charge transfer results in smear in the output image (Bell et al. 2006). The image smear is usually removed by a certain image processing algorithm (Powell et al. 1999). MMS uses a special band to directly measure smear for removal during ground processing. This band is located at the last 3 readout lines at the edge of the CCD, 
Fig. 26 The radiometric calibration coefficients ratio of short and long integration time

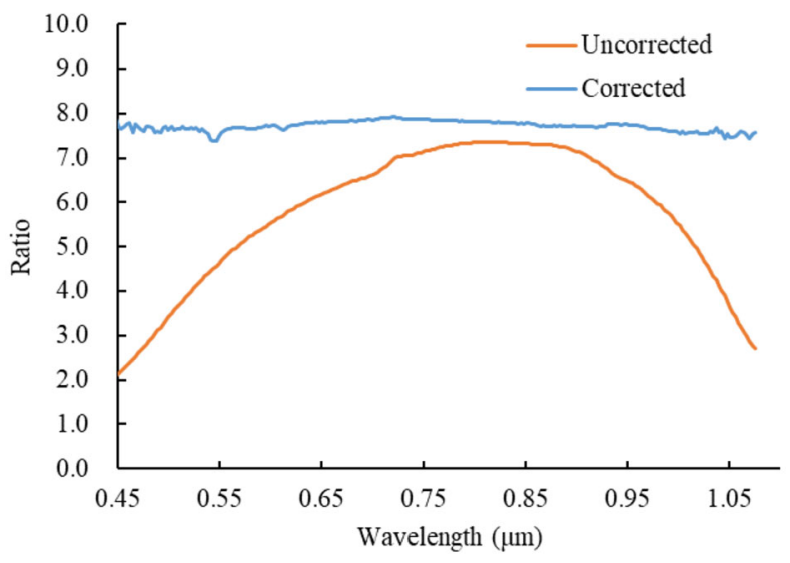

which could not be illuminated by light from either the scene or calibration unit so the readout reflects completely the magnitude of the smear. The smear data is retrieved at the 256th-band in the original readout cube of the CCD. The authors removed image smear by subtracting the smear channel signal from the measured dark-current-removed signal value. The calculation method is given by:

$$
\begin{aligned}
\breve{S}_{i, j, k, t} & =\widehat{S}_{i, j, k, t}-\widehat{S}_{i, j, 255, t} \quad(k=0,2,3, \ldots, 254), \\
\widehat{S}_{i, j, k, t} & =S_{i, j, k, t}-\bar{D}_{i, k, t} \quad(k=0,2,3, \ldots, 254), \\
\bar{D}_{i, k, t} & =\frac{1}{N_{L, D B}} \sum_{j=1}^{N_{L, D B}} D_{i, j, k, t} \quad(k=0,2,3, \ldots, 254),
\end{aligned}
$$

where $\breve{S}_{i, j, k, t}$ is the smear-removed signal value; $i, j, k$ and $t$ is the serial number of sample, line, band, and energy level of a hyperspectral data cube, respectively; $\widehat{S}_{i, j, k, t}$ is the darkcurrent-removed signal value; and $\widehat{S}_{i, j, 255, t}$ is the smear signal value; $S_{i, j, k, t}$ is the DN value of the raw data cube; $\bar{D}_{i, k, t}$ is the dark background after time dimension averaging; $D_{i, j, k, t}$ is the DN value of measured dark background; and $N_{L, D B}$ is number of lines of measured dark background data.

Theoretically, the ratio of the radiometric calibration coefficients between the long integration time $(44.635 \mathrm{~ms})$ and the short integration time $(5.155 \mathrm{~ms})$ should be around 8.6 for all V-NIR bands. Even if the ratio is not around 8.6, it fluctuates at least around a constant. The ratios before smear removal vary significantly with bands, and the maximum value is more than 3 times the minimum value in $0.45-1.05 \mu \mathrm{m}$. Whereas the ratios after smear removal fluctuate within a range of $7.7 \pm 0.1$ (mean \pm standard deviation), which is in line with the actual situation. The results (Fig. 26) show that after smear correction, the effect of smear on the signal values is mostly eliminated.

\subsubsection{Non-uniformity Correction}

For a linear-array push-broom imaging spectrometer, the response of different pixels in the same band in a row to a uniform incident radiation is often inconsistent (Zhou et al. 2005). A non-uniformity correction is primarily to eliminate this inconsistency and perform normalization to remove pixel-to-pixel response non-uniformity. The obtained non-uniformity 
a)

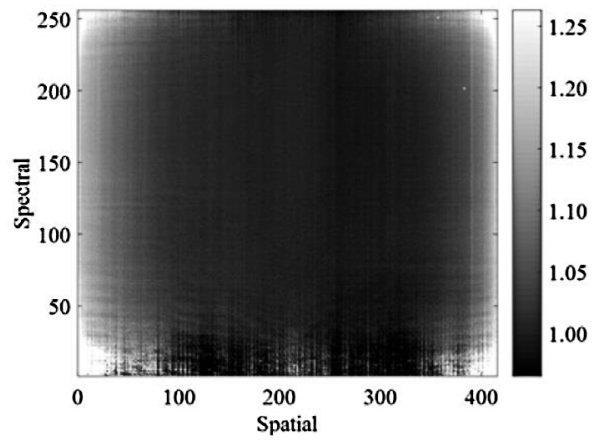

b)

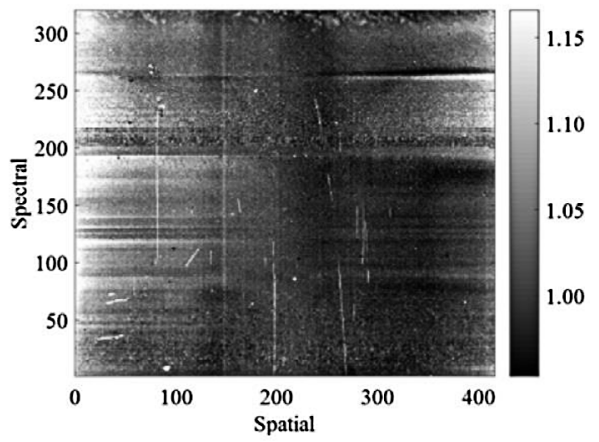

Fig. 27 Non-uniformity correction coefficient: a) V-NIR region (integration time of $44.635 \mathrm{~ms}$ ). b) N-MIR region (integration time of $23.04 \mathrm{~ms}$ )

correction coefficient is also called relative radiometric calibration coefficient. For the nadir observation region on detectors, two-point method of the non-uniformity correction of has been taken by measure the uniform reference source such as integrating sphere and area blackbody. Certainly, the dark background estimation and smear correction need be subtracted first. The calculation method of non-uniformity correction coefficient (Mudau et al. 2011) is as follows:

$$
\begin{aligned}
\bar{S}_{k, t} & =A_{i, k} \times \bar{S}_{i, k, t} \quad(i=0,2,3, \ldots, 415), \\
\bar{S}_{i, k, t} & =\frac{1}{N_{L}} \sum_{j=1}^{N_{L}} \breve{S}_{i, j, k, t}, \\
\overline{\bar{S}}_{k, t} & =\frac{1}{N_{S}} \sum_{i=1}^{N_{S}} S_{i, k, t},
\end{aligned}
$$

where $\breve{S}_{i, j, k, t}$ is the signal value after the dark background is subtracted from the original data cube (smear removal in the V-NIR region is also performed); $\bar{S}_{i, k, t}$ is the signal value of a certain pixel in a certain band; $\overline{\bar{S}}_{k, t}$ is the standard reference value of all pixels in a band (the authors took the average value of the main field of view of pixels 161-260); $A_{i, k}$ is the non-uniformity correction coefficient; $N_{L}$ is the total number of lines; $N_{S}$ is the total number of samples, that is, the number of pixels in each band for the imaging area, here is 416. According to the measured data under different energy level, the non-uniformity correction coefficient was calculated using the least-squares method. During the image data processing, the derived non-uniformity correction coefficient matrix can be used to perform non-uniformity correction on the data to be corrected.

Figure 27 shows the non-uniformity correction coefficients in the V-NIR and N-MIR regions, respectively. It can be seen that most of the coefficients $\left(A_{i, k}\right)$ are around 1.0. This aspect shows that after the dark current is subtracted, except for the individual pixels, the signal value shift caused by the dark current is mostly eliminated. Through non-uniformity correction, the uniformity of response of each detection unit of the sensor to the entrance pupil radiation can be greatly improved. Because $A_{i, k}$ is more relevant when measuring the response inconsistency of different pixels on the focal plane, the textures of two-dimensional images composed of $A_{i, k}$ coefficients with different integration time are very similar. 

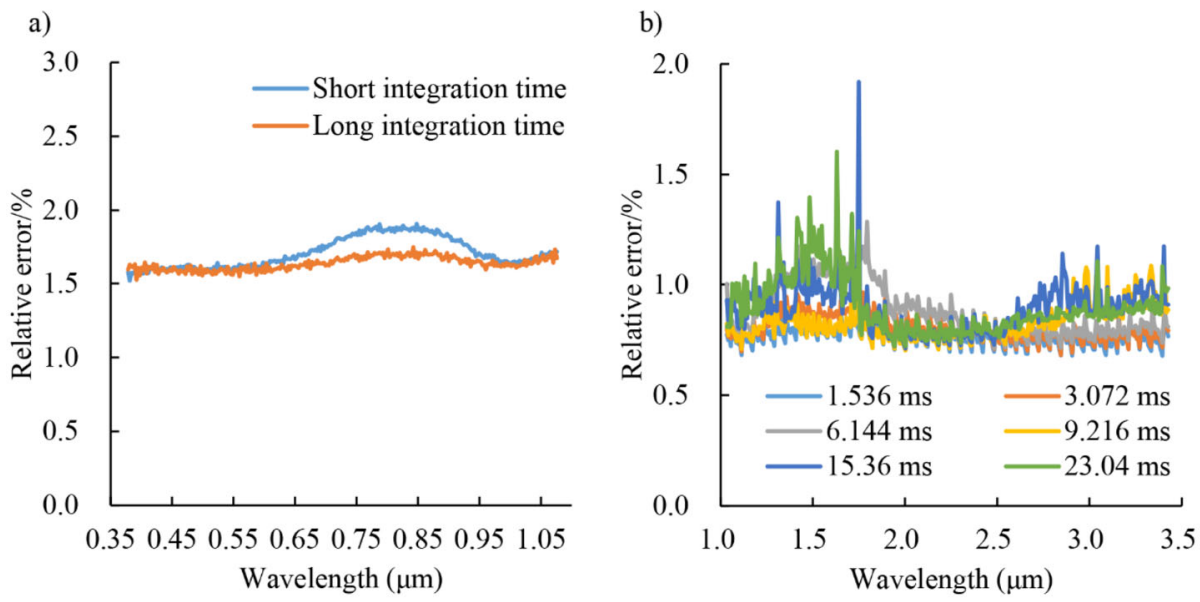

Fig. 28 Errors of non-uniformity correction a) V-NIR region; b) N-MIR region

Figure 28 shows the error of non-uniformity correction in the V-NIR and N-MIR regions, respectively. The error of the non-uniformity correction model is within $2.0 \%$ in both V-NIR region and N-MIR region. The main reason for the error is, that the strip noise in spatial dimensional, caused by the inconsistent response of detection unit, has been largely eliminated, and the remaining represents only a small amount of random noise.

\subsubsection{Absolute Radiometric Calibration}

The process of absolute radiometric calibration is generally to establish a model that can well describe the instrument's response to radiance (Murchie et al. 2007; Chander et al. 2009). By acquiring MMS data cube of reference light sources (integrating sphere and black body) with different energy levels, combined with the known radiance spectra of reference light sources with different energy levels, the relationship between MMS input and output can be determined, and absolute radiometric calibration can be achieved. After comparing the effects of linear, logarithm, exponent and quadratic function, the quadratic function was chosen at last. The formula used for the absolute radiometric calibration of MMS can be expressed as

$$
L_{k, t}=a_{k} \times \overline{\bar{S}}_{k, t}^{2}+b_{k} \times \overline{\bar{S}}_{k, t}+c_{k},
$$

where $L_{k, t}$ is the radiance; $a_{k}, b_{k}$ and $c_{k}$ is the absolutely radiometric calibration coefficient, respectively. In a laboratory calibration setup using a reference light source, $L_{k, t}$ is the integration of the spectral response function and the radiance spectrum of the reference light source. The calculation method is given by

$$
L_{k, t}=\frac{\int s r f_{k}(\lambda) t_{w i n}(\lambda) L_{t}(\lambda) d \lambda}{\int s r f_{k}(\lambda) d \lambda},
$$

where $\lambda$ is the wavelength; $L_{t}(\lambda)$ is the radiance of the reference light source at the energy level $t ; s r f_{k}(\lambda)$ is the spectral response function of band $k$; and $t_{w i n}(\lambda)$ is the window transmittance of the cryogenic box used in radiometric calibration. Based on the measured data 
a)

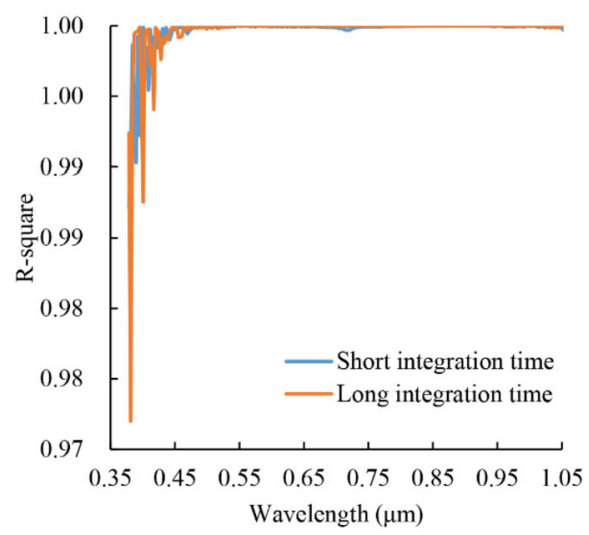

b)

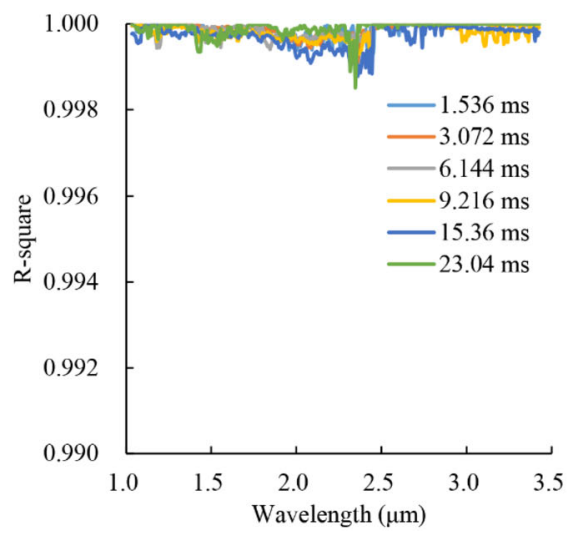

Fig. 29 Coefficient of determinations in a) V-NIR region and b) N-MIR region

a)

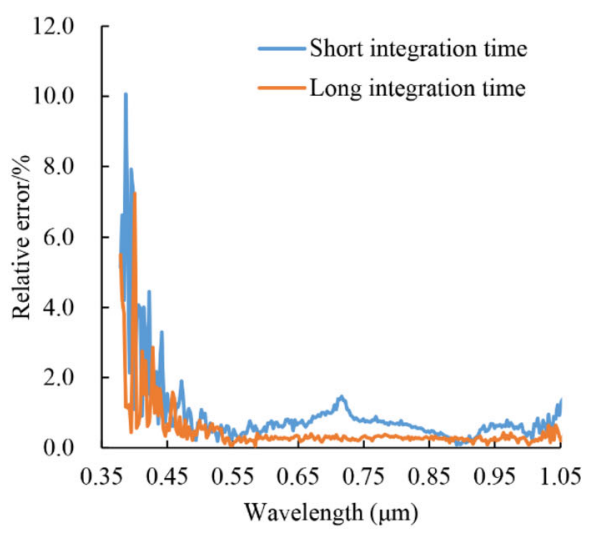

b)

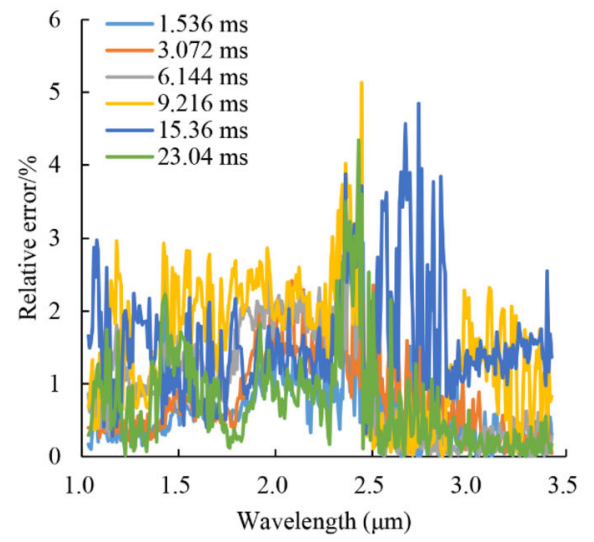

Fig. 30 Absolutely radiometric calibration errors of various integration times in the V-NIR region a) and N-MIR channel b)

and the radiance spectrum of the integrating sphere, the coefficients are estimated using the least squares method.

Figure 29 shows the coefficient of determinations (R-squares) of radiometric calibration coefficients for each integration time in the V-NIR region and N-MIR region, respectively. The average R-squares for each integration time sensor is above 0.99 , indicating that the calibration equation worked well. Figure 30 shows the error curves of absolutely radiometric calibration in the V-NIR and N-MIR regions, respectively. The maximum error of the short integration time is approximately $10.1 \%$ and the maximum error of the long integration time is approximately $5.1 \%$. The slightly worse performance of the R-squares and the calibration errors in short wavelengths of V-NIR region is probably due to the low irradiance in of the integrating sphere in those wavelengths. The similar phenomena can be also found around $2.5 \mu \mathrm{m}$ in N-MIR region. 
Fig. 31 The $\mathrm{S} / \mathrm{N}$ of the MMS (at $1.52 \mathrm{AU}$, phase angle of $45^{\circ}$, and albedo of $15 \%$ )

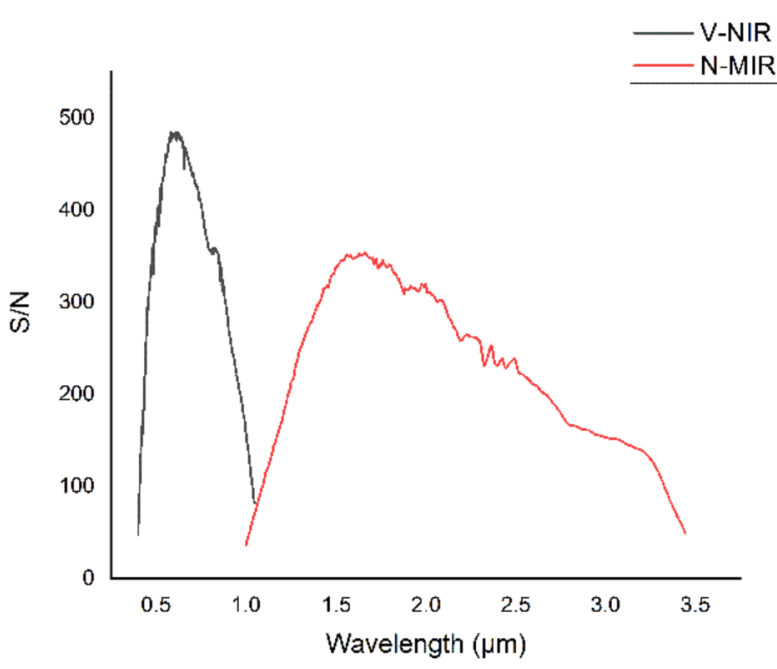

Table 11 Measurement results of internal orientation elements

\begin{tabular}{lll}
\hline Parameter & V-NIR & N-MIR \\
\hline $\begin{array}{l}\text { Principal point } \\
\text { position }\end{array}$ & Sample 207 & Sample 205 \\
$\begin{array}{l}\text { Principal } \\
\text { distance (mm) }\end{array}$ & 51.940 & 49.942 \\
IFOV (mrad) & 0.501 & 0.502 \\
TFOV $\left(^{\circ}\right)$ & 11.950 & 11.981 \\
Lens distortion & $\Delta y=-8.814 \mathrm{e}-5 i^{2}+0.03989 i-4.547$ & $\Delta y=-8.776 \mathrm{e}-5 i^{2}+0.03959 i-4.416$ \\
\hline
\end{tabular}

Based on the optical specification and radiation response, the signal-to-noise ratio of MMS is shown in Fig. 31, modeled with $1.52 \mathrm{AU}$ illumination, phase angle of $45^{\circ}$, albedo of 0.15 , and Martian surface temperature of $250 \mathrm{~K}$.

\subsubsection{Geometric Calibration}

In order to ensure measurement accuracy, it is necessary to calibrate the internal orientation elements of the spectral imager accurately. If high-precision spectral calibration is used to solve the problem of spectral positioning of MMS, then high-precision internal orientation element calibration is used to solve the problem of geometric positioning of MMS, which belongs to the problem of spatial dimension.

Table 11 shows the results of geometric calibration. The principal point positions are samples 207 and 205 for V-NIR and N-MIR, respectively, which is essentially the centre of the imaging region (samples 0-415) of MMS. The principal distance, IFOV, and TFOV are also similar for V-NIR and N-MIR. The characteristics of lens distortion show a parabola with the vertex around the principal point for both V-NIR and N-MIR. The similarity in internal orientation elements indicates that MMS can be obtained the data cube with similar spatial resolution and swath in V-NIR and N-MIR regions, which is convenient for the registration of the data cubes of V-NIR and N-MIR in a high accuracy. Table 12 lists the 
Table 12 Matching measurement between observation coordinate system of V-NIR and N-MIR channels

\begin{tabular}{llrrr}
\hline Channel & $\begin{array}{l}\text { Observation } \\
\text { coordinate system }\end{array}$ & \multicolumn{4}{l}{$\begin{array}{l}\text { Observation coordinate system } \\
\text { (V-NIR channel) }\end{array}$} \\
\cline { 3 - 5 } & & $\mathrm{Xv}$ & $\mathrm{Yv}$ & $\mathrm{Zv}$ \\
\hline \multirow{2}{*}{ N-MIR } & $\mathrm{Xn}$ & 0.0093 & 89.9938 & 89.9930 \\
& Yn & 90.0062 & 0.0063 & 90.0009 \\
& $\mathrm{Zn}$ & 89.9931 & 89.9991 & 0.0070 \\
\hline
\end{tabular}

registration relationship between the observation coordinate system of two channels, which expressed axis direction in Fig. 1. Z-axis of observation coordinate system is along the direction of the principal point position's IFOV, and the Y-axis is along the slit direction.

During data preprocessing in MMS, the geometric distortion correction of data is mainly to correct the lens distortion of the acquired data cube in the push-broom direction, that is, the distortion perpendicular to the spatial dimension. Combined with the geometric lens distortion characteristics of MMS, the direct interpolation method is used to perform pixel coordinate conversion. According to the calculated pixel coordinates of the output image, the parameters for V-NIR and N-MIR can be referred to in Table 11 respectively. Then select the integer coordinate pixels of the output image, and select a few points (such as two) that are closest to the integer coordinates to linearly interpolate in order to obtain the integer coordinate pixel values.

\subsection{In-flight Calibration}

When MMS is in its remote sensing orbit, the blocked area of the slits is used to monitor the changes in dark background driven by variation in the temperature of the OSU (Widenhorn et al. 2002; Kuusk 2011; Sizov et al. 2005). The data from corresponding blocked pixels can be used to estimate the original dark background DN value of other pixels, based on the dark current model acquired pre-flight or observed looking at deep space in-flight. In each operation mode, the dark current reference is collected synchronously and packaged in the scientific data; the dark background of the nadir observation area is estimated based on the full-frame dark background data and the DN value relationship of the blocked samples. The estimation process is as follows:

(1) Dark background average and smoothing. In order to reduce the effect of noise on dark background estimation, the dark background monitoring area is averaged spatially and smoothed temporally (row direction).

(2) Dark background estimation and elimination. The essential component of dark background in V-NIR region of MMS is dark current. However, in addition to the dark current of the detector, the internal background radiation of the instrument is also an important source of dark background in N-MIR region; when there is an increase in the internal temperature of the instrument, the internal background radiation will increase rapidly. Two different methods were adopted for V-NIR and N-MIR regions. For the VNIR region, a method of adjusting according to a scale factor was used. For the N-MIR region, a linear model was used.

For the absolute radiometric calibration, the CU includes built-in integrating sphere and solar viewer, providing in-flight calibration capability for MMS to monitor the stability of its radiance response. In the Earth-Mars transfer orbit, MMS will conduct a monthly self-test to compare the response data with pre-launch data by lighting up the lamps of the built-in 

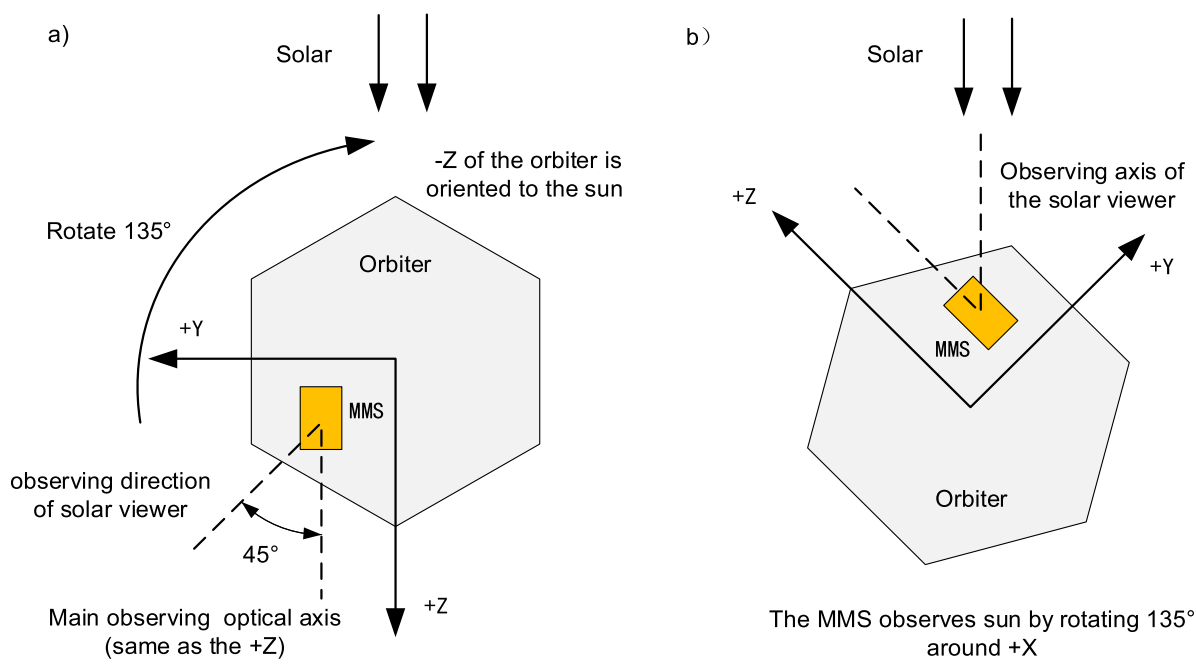

The MMS observes sun by rotating $135^{\circ}$ around $+X$

Fig. 32 The attitude of the orbiter's Sun orientation mode and MMS' solar observation mode

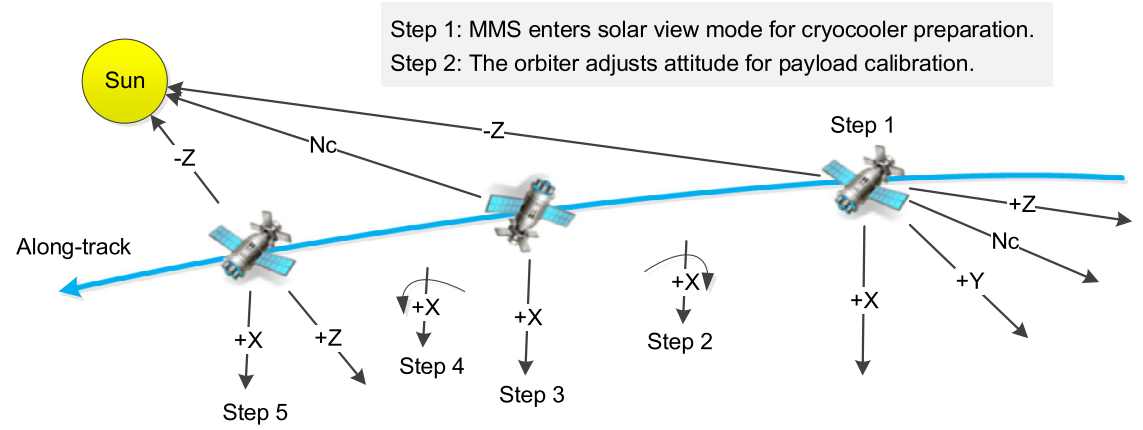

Step 3: After the attitude is stable, the solar viewer points towards the sun and the calibration data will be acquired. Step 4: The orbiter adjusts attitude for sun-oriented mode.

Step 5: After the attitude is stable, the MMS looks away from the sun and space observation will be operated.

Fig. 33 The in-flight calibration schedule of MMS

integrating sphere. When the orbiter reaches the remote sensing orbit, the solar and space observations will continue monthly. The orbiter will maintain the Sun-oriented mode $(-\mathrm{Z}$ axis pointing to the Sun) above $800 \mathrm{~km}$ and will obtain electrical energy through its solar panels. The solar viewer of MMS observes at an angle of $45^{\circ}$ from the main optical axis of the OSU, which is parallel to the $+\mathrm{Z}$ axis. The orbiter will conduct attitude adjustment of $135^{\circ}$ around the $+\mathrm{X}$ axis so that the solar viewer of MMS is aligned to the Sun, which is described in Fig. 32. The orbiter will maintain the payload calibration attitude for 5 minutes to ensure data acquisition. After the solar observation is completed, the orbiter will return to the Sun-oriented mode. At this time, the main FOV of MMS will be turned away from the Sun, and space observation can be implemented, which is used to obtain the full-frame dark current image of the two detectors in-orbit. The whole schedule is shown in Fig. 33. 


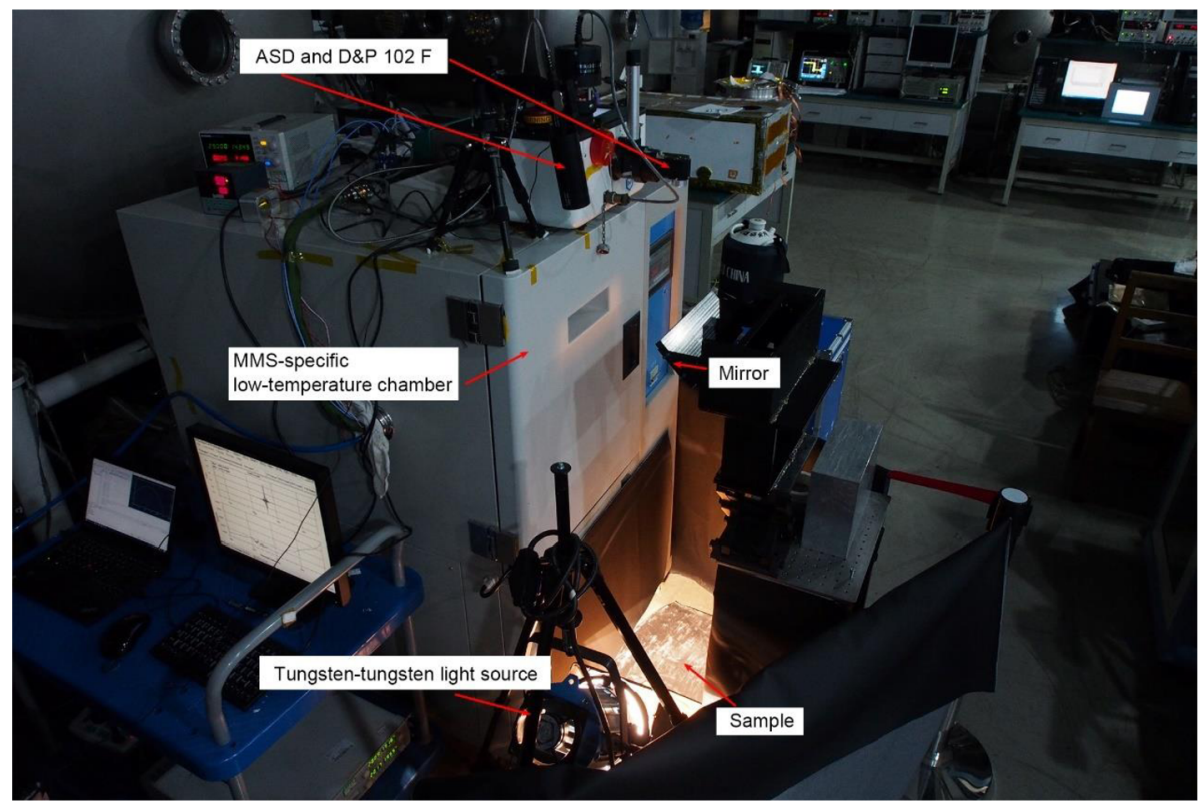

Fig. 34 The MMS's ground verification test used the EQM in a laboratory environment. The EQM was placed in a low-temperature chamber with sapphire window glass and internal nitrogen filling. The powder samples placed on the ground were observed by mirror outside the chamber. An ASD FieldSpec-4 and D\&P $102 \mathrm{~F}$ were used as references

\section{Laboratory Data}

In the ground verification test carried out by SITP and NAO, the EQM of MMS instrument performed spectral observation tests on a variety of mineral samples, such as smectite, hypersthene, goethite, chlorite, apatite, etc. Selected laboratory test data are shown in Fig. 34, where several samples and standard reflectance panels (Labsphere Inc.) were illuminated with the light source of halogen lamp. The samples were measured with MMS and commercial spectrometers (ASD FieldSpec-4 and D\&P $102 \mathrm{~F}$ ). In this test, a $600 \mathrm{~W}$ halogen lamp was used as irradiate a light source to illuminate the reflectance panels and the samples of mineral powders with an incident angle of 45 degrees. The EQM was placed in a low-temperature chamber with sapphire window glass and internal nitrogen filling.

The reflectance factor (REFF) of samples were calculated and joined according to the spectral radiance measured by MMS, and the spectra of the powder samples were converted to REFF by normalizing by spectra of the Spectrolon standard panels at $0.35-2.5 \mu \mathrm{m}$, and the Infragold one at 2.5-3.4 $\mu \mathrm{m}$. The joined method of the overlapping spectra between two detectors can be described as follows: taking the N-MIR channel as reference, comparing the first derivative of the spectra to find the most similar part in the overlapping spectral range (1.03-1.07 $\mu \mathrm{m})$, and calculating the compensation coefficient of the V-NIR spectrum. Figure 35a shows the REFF of the mineral powder samples acquired using the D-26 operation mode of MMS with high spectral resolution. Hypersthene is a typical silicate mineral with broadband absorption characteristics. Figure 35b compares the measurement results of the hypersthene sample in different operation modes. All 6 multispectral band sets can describe the spectral characteristic as well. Among them, a versatile set (multispectral set 1) 
a)

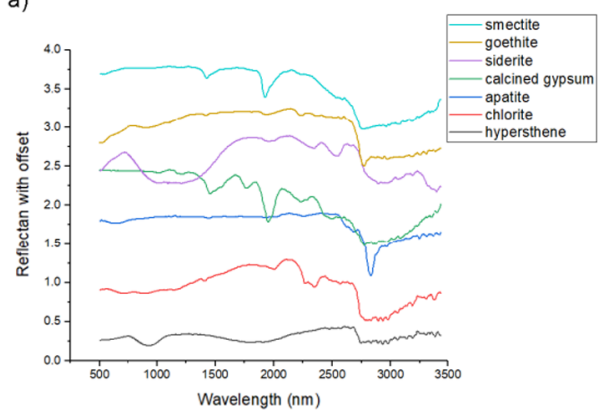

b)

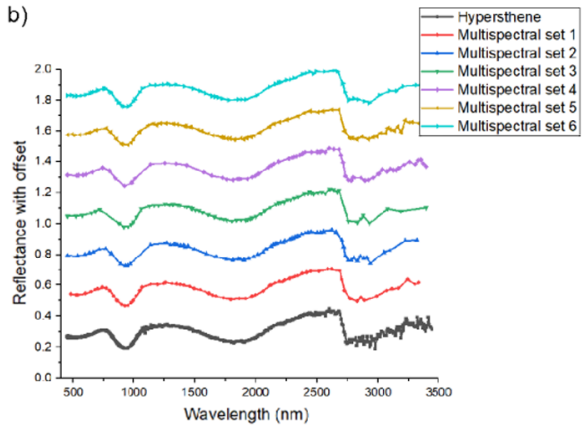

Fig. 35 a) The REFF of monomineralic powder samples obtained by the EQM of MMS in the ground verification test, offset of 0.5 is added to distinguish. b) The REFF of hypersthene obtained by the EQM of MMS used MMS-D-26 mode, and MMS-D-208 mode with 6 different multispectral band sets, offset of 0.5 is added to distinguish

has more uniform sampling distribution for the key absorption positions near $1.0 \mu \mathrm{m}$ and $2.0 \mu \mathrm{m}$.

\section{Summary and Conclusions}

This paper describes the design, operation, and performance of MMS, a multifunctional imaging spectrometer onboard the Tianwen-1 Mars exploration mission of China. MMS consists of (1) an Optical Sensor Unit, which performs observations with spectral response coverage at $0.4-3.4 \mu \mathrm{m}$, with a $0.5 \mathrm{mrad}$ IFOV and $12^{\circ}$ TFOV; (2) a Calibration Unit, which provides a built-in integrating sphere and solar viewer; (3) an Electronics Unit; and (4) a Thermal Control Accessories. With operation modes such as multi-sample hyperspectral mode and multispectral imaging mode, MMS will perform nadir observation in the remote sensing orbit when the orbital height is less than $800 \mathrm{~km}$. Spectral data will be used to identify the minerals on the Martian surface including, most importantly, hydrated minerals and water ice. MMS has completed flight mode tests, in which all performance requirements have been verified. Through data accumulation during its lifetime of one Martian year, MMS is expected to contribute to new scientific discoveries in the near future.

Acknowledgements This work was supported by China's first Mars exploration program and China National Space Administration (CNSA), and was also funded by National Natural Science Foundation of China (NSFC) (Grant No. 21105109, No. 61605231, and No. U1931211), Youth Innovation Promotion Association CAS (No. 2016018, and No. 2017286), and the Program of Shanghai Academic/Technology Research Leader (No. 19XD1424100). The MMS team wishes to thank the other researchers and colleagues who supported the MMS program at various institutes or academies, including China Academy of Space Technology (CAST), National Astronomical Observatories of China (NAOC), National Space Science Centre (NSSC), Kunming Institute of Physics (KIP), and Xidian University. Assistance at SITP by Haimei Gong, Rong Shu, Xue Li, Songmin Zhou, Jianfei Kang, Yuanting Shen, Dingzhen Liu, Binyong Wang, Yaoying Guo, Shuming Zhang, Hao Jiang, Ruijun Ding, Yinong Wu, Jinwen Lu, Lin Wu, Libin Ye, Quanquan Zhang, Xuemin Liu, Ruxin Xu, Jianjun Jia, Baolong Zhang, Xiaopeng Liu, Minsheng Cheng, Yuquan Zhang, Zhikang Bao, Bo Zhou, Liqing Wang is greatly appreciated.

Author Contributions Study conception and design were performed by Zhiping He, Jianyu Wang, Chunlai Li, Gang Lv, Liyin Yuan, and Rui Xu. Material preparation, engineering development, data collection, and analysis were performed by Zhiping He, Chunlai Li, Gang Lv, Rui Xu, Liyin Yuan, Jian Jin, Chengyu Liu, Jianan Xie, Chuifeng Kong, Feifei Li, Xiaowen Chen, Rong Wang, Sheng Xu, Wei Pan, Jincai Wu, Changkun 
Li, Tianhong Wang, Haijun Jin, Hourui Chen, and Jun Qiu. The manuscript was written and edited by Zhiping He, Rui Xu, Chengyu Liu, Liyin Yuan, Chunlai Li, Gang Lv, Jian Jin, and Jianan Xie. This work was supervised by Zhiping He, the Principal Investigator of this project. All authors read and approved the final manuscript.

Conflicts of Interest The authors declare that they have no conflicts of interest.

Publisher's Note Springer Nature remains neutral with regard to jurisdictional claims in published maps and institutional affiliations.

Open Access This article is licensed under a Creative Commons Attribution 4.0 International License, which permits use, sharing, adaptation, distribution and reproduction in any medium or format, as long as you give appropriate credit to the original author(s) and the source, provide a link to the Creative Commons licence, and indicate if changes were made. The images or other third party material in this article are included in the article's Creative Commons licence, unless indicated otherwise in a credit line to the material. If material is not included in the article's Creative Commons licence and your intended use is not permitted by statutory regulation or exceeds the permitted use, you will need to obtain permission directly from the copyright holder. To view a copy of this licence, visit http://creativecommons.org/licenses/by/4.0/.

\section{References}

W.H. Bakker, F.J.A. van Ruitenbeek, H.M.A. van der Werff et al., Processing OMEGA/Mars Express hyperspectral imagery from radiance-at-sensor to surface reflectance. Planet. Space Sci. 90, 1-9 (2014)

J.F. Bell, J. Joseph, J.N. Sohl-Dickstein, H.M. Arneson, M.J. Johnson, M.T. Lemon, D. Savransky, J. Geophys. Res. 111(2), E02S03 (2006)

E. Belyaev, K. Liu, M. Gabbouj, Y.S. Li, IEEE Trans. Circuits Syst. Video Technol. 25(8), 1435 (2015)

J.P. Bibring, Y. Langevin, A. Gendrin, B. Gondet, F. Poulet et al., Mars surface diversity as revealed by the OMEGA/Mars Express observations. Science 307, 1576-1581 (2005)

J.P. Bibring, Y. Langevin, J.F. Mustard, F. Poulet, R. Arvidson et al., Global mineralogical and aqueous Mars history derived from OMEGA/Mars Express data. Science 312, 400-404 (2006)

K.O. Boltar, L.A. Bovina, L.D. Saginov, V.I. Stafeev, I.S. Gibin, V.M. Maleev, IR imager based on a $128 \times 128 \mathrm{HgCdTe}$ staring focal plane array, in Proc. SPIE 3819, International Conference on Photoelectronics and Night Vision Devices, 10 June 1999 (1999). https://doi.org/10.1117/12.350889

G. Bonello, J.P. Bibring, F. Poulet et al., Visible and infrared spectroscopy of mineral and mixtures with the OMEGA/MARS-EXPRESS instrument. Planet. Space Sci. 52(1), 133-140 (2004)

G. Chander, B.L. Markham, D.L. Helderc, Remote Sens. Environ. 113(5), 893 (2009)

L.G. Cook, J.F. Silny, Imaging spectrometer trade studies: a detailed comparison of the Offner-Chrisp and reflective triplet optical design forms. Proc. SPIE 7813, 78130F (2010)

J. Dong, Z. Sun, W. Rao, Y. Jia, L. Meng, C. Wang, B. Chen, Mission profile and design challenges for Mars Landing exploration, in The International Archives of the Photogrammetry, Remote Sensing and Spatial Information Sciences, Volume XLII-3/W1, Proc. 2017 International Symposium on Planetary Remote Sensing and Mapping, Hong Kong, 13-16 August 2017 (2017), pp. 13-16. https://doi.org/10.5194/ isprs-archives-XLII-3-W1-35-2017

J. Hou, Z. He, R. Shu, Optical design of 400-1000 nm spectral imaging system based on a single freeform mirror, in Proc. SPIE 9678, AOPC 2015: Telescope and Space Optical Instrumentation, Beijing, China, 8 October 2015 (2015). https://doi.org/10.1117/12.2199371

Y.Z. Jia, Y. Fan, Y.L. Zou, Chin. J. Space Sci. 38(5), 650 (2018). https://doi.org/10.11728/cjss2018.05.650

J. Kuusk, J. Sens., 608157 (2011)

C. Li, J. Liu, Y. Geng, J. Cao, T. Zhang, G. Fang, J. Yang, R. Shu, Y. Zou, Y. Lin, Z. Ouyang, J. Deep Space Explor. 5(5), 406 (2018)

D. Liu, X. Ren, H. Zhang, Z. Zhang, Q. Fu, Z. He, B. Liu, R. Xu, Y. Chen, Preliminary wavelengths selection for multispectral detection mode of Mars Mineral Spectrometer of China's First Mars Exploration, in EPSC Abstracts, EPSC2018-574-2 vol. 12 (2018)

B. Liu, X. Ren, D. Liu et al., Ground validation experiment and spectral detection capability evaluation of Mars Mineralogical Spectrometer (MMS) aboard on HX-1 orbiter. Space Sci. Rev. (2021, forthcoming), Manuscript Number: SPAC-D-21-00007

P.Z. Mouroulis, R.G. Sellar, D.W. Wilson, J.J. Shea, R.O. Green, J. Geophys. Opt. Eng. 46(6), 063001 (2007) 
A.E. Mudau, C.J. Willers, D. Griffith, F.P. Roux, Non-uniformity correction and bad pixel replacement on LWIR and MWIR images, in Proc. IEEE Saudi International Electronics, Communications and Photonics Conference (SIECPC), Riyadh, Saudi Arabia, 24-26 April 2011 (2011), pp. 1-5

S.L. Murchie, R.E. Arvidson, P.D. Bedini, K. Beisser, J.-P. Bibring, J. Boldt et al., Compact Reconnaissance Imaging Spectrometer for Mars (CRISM) on Mars Reconnaissance Orbiter (MRO). J. Geophys. Res. 112, E05S03 (2007). https://doi.org/10.1029/2006JE002682

S.L. Murchie, F.P. Seelos, C.D. Hash et al., Compact Reconnaissance Imaging Spectrometer for Mars investigation and data set from the Mars Reconnaissance Orbiter's primary science phase. J. Geophys. Res. 114, E00D07 (2009). https://doi.org/10.1029/2009JE003344

K. Powell, D. Chana, D. Fish, C. Thompson, Appl. Opt. 38(8), 1343 (1999)

F.F. Sizov, J.V. Gumenjuk-Sichevska, I.O. Lysiuk, V.V. Zabudsky, A.G. Golenkov, S.G. Bunchuk, Temperature dependence of the dark current in HgCdTe photodiode arrays, in Proc. SPIE 5957, Infrared Photoelectronics (2005), 59571L

Teledyne e2v (UK) Limited, CCD47-20 Back Illuminated NIMO Frame-Transfer High Performance CCD Sensor (2017). https://www.teledyne-e2v.com/products/space/ccd-image-sensors-for-space-andground-based-astronomy/

R. Widenhorn, M.M. Blouke, A. Weber, A. Rest, E. Bodegom, in Proceedings Volume 4669, Sensors and Camera Systems for Scientific, Industrial, and Digital Photography Applications III, Electronic Imaging, San Jose, California, United States (2002). https://doi.org/10.1117/12.463446

R. Xu, Z.P. He, H. Zhang, Y.H. Ma, Z.Q. Fu, J.Y. Wang, Calibration of imaging spectrometer based on acousto-optic tunable filter (AOTF), in Proc. SPIE Asia-Pacific Remote Sensing. International Society for Optics and Photonics (2012), pp. 85270S-85270S

R. Xu, G. Lv, Y.H. Ma, J.Y. Wang, Calibration of Visible and Near-infrared Imaging Spectrometer (VNIS) on lunar surface. Proc. SPIE 9263, 926315 (2014)

P.J. Ye, Z.Z. Sun, W. Rao, L.Z. Meng, Sci. China, Technol. Sci. (2017). https://doi.org/10.1007/ s11431-016-9035-5

L.Y. Yuan, Z.P. He, Y.J. Wang, G. Lv, Manufacture, alignment and measurement for a reflective triplet optics in imaging spectrometer. Proc. SPIE 9684, 96840B (2016)

L.Y. Yuan, Z.P. He, G. Lv, Y. Wang, C.L. Li, J.N. Xie, J.Y. Wang, Opt. Express 25(19), 22440 (2017)

L.Y. Yuan, J.N. Xie, J. Hou, G. Lv, Z.P. He, Infrared Laser Eng. 47(4), 0418001 (2018)

L.Y. Yuan, J.N. Xie, Z.P. He, Y.J. Wang, J.Y. Wang, Opt. Express 27(13), 17686-17700 (2019)

H. Zhou, S. Liu, R. Lai, D. Wang, Appl. Opt. 44(15), 2928 (2005) 